\title{
Optimal Fuzzy Controller Design in Continuous Fuzzy System: Global Concept Approach
}

\author{
Shinq-Jen Wu and Chin-Teng Lin, Senior Member, IEEE
}

\begin{abstract}
In this paper, we propose a systematic and theoretically sound way to design a global optimal fuzzy controller to control and stabilize a continuous fuzzy system with free- or fixed-end point under finite or infinite horizon (time). A linear-like global system representation of continuous fuzzy system is first proposed by viewing a continuous fuzzy system in global concept and unifying the individual matrices into synthetical matrices. Based on this, the optimal control law which can achieve global minimum effect is developed theoretically. The nonlinear segmental two-point boundary-value problem (TPBVP) is derived for the finite-horizon problem and a forward Riccati-like differential equation (DE) for the infinite-horizon problem. To further simplify the computation, a segmental Riccati-like DE is derived in solving the finite- or infinite-horizon issues. Moreover, in the case of time-invariant fuzzy systems, we show that the optimal controller can be obtained by just solving algebraic Riccati-like equations. Grounding on this, several fascinating characteristics of the resultant closed-loop fuzzy system can be elicited easily. The stability of the closed-loop fuzzy system can be ensured by the designed optimal fuzzy controller. The optimal closed-loop fuzzy system cannot only be guaranteed to be exponentially stable, but also be stabilized to any desired degree. Also, the total energy of system output is absolutely finite. Moreover, the resultant closed-loop fuzzy system possesses an infinite gain margin; that is, its stability is guaranteed no matter how large the feedback gain becomes. An example is given to illustrate the proposed optimal fuzzy controller design approach and to demonstrate the proved stability properties.
\end{abstract}

Index Terms-Degree of stability, finite energy, global minimum, Riccati-like equation, two-point boundary-value problem (TPBVP).

\section{INTRODUCTION}

A LTHOUGH the research in fuzzy modeling and fuzzy control has been quite matured [1]-[9], it seems that the field of optimal fuzzy control is nearly open [10]. The goal of this work is to propose a systematic and theoretically sound scheme for designing a global optimal fuzzy controller to control and stabilize a continuous-time fuzzy system with free- or fixed-end point under finite or infinite horizon.

Manuscript received December 7, 1999; revised June 19, 2000. This work was supported by the R.O.C. National Science Council under Grant NSC892622-E-009-005.

S.-J. Wu is with the Department of Electrical Engineering, Da-Yeh University, Chang-Hwa, Taiwan 515, R.O.C.

C.-T. Lin is with the Department of Electrical and Control Engineering, National Chiao-Tung University, Hsinchu, Taiwan 300, R.O.C. (e-mail: ctlin@fnn.cn.nctu.edu.tw).

Publisher Item Identifier S 1063-6706(00)10694-0.
Stability and optimality are the most important requirements for any control system. Most of the existed works on the stability analysis of fuzzy control are based on Takagi-Sugeno (T-S) type fuzzy model combined with parallel distribution compensation (PDC) concept [1] and apply Lyapunov's method to do stability analysis. Tanaka et al. [2], [4] reduced the stability analysis and control design problems to linear matrix inequality (LMI) problems. They also dealt with uncertainty issue [3]. This approach had been applied to several control problems such as control of chaos [4] and of articulated vehicle [6]. A frequency shaping method for systematic design of fuzzy controllers was also done by them [11]. Sun et al. [7] developed a separation scheme to design fuzzy observer and fuzzy controller independently. Methods based on grid-point approach [12] and circle criteria [13], [14] were introduced to do stability analysis of fuzzy control, too. Wang adopted a supervisory controller and introduced stability and robustness measures [15]. Cao proposed a decomposition principle to design a fuzzy discrete-time control system and an equivalent principle to do stability analysis [9]. Even with the aforementioned research results on the theoretic aspect of fuzzy control, Tanaka and others' works mentioned in the above always treat the stability of general linear feedback fuzzy controllers.

On the issue of optimal fuzzy control, Wang developed an optimal fuzzy controller to stabilize a linear time-invariant system via Pontryagin maximum principle [10]. However, although fuzzy control of linear systems could be a good starting point for a better understanding of some issues in fuzzy control synthesis, it does not have much practical implications since using the fuzzy controller designed for a linear system directly as the controller may not be a good choice [10]. Moreover, the cited stability criteria may be simple, but rough to do systematic analysis and also may result in a controller with less flexibility. Tanaka et al. [5], [16], [17] tried to obtain a fuzzy controller to minimize the upper bound of the quadratic performance function by the LMI approach based on the assumption of local-linear-feedback-gain control structure. Nevertheless, no theoretical analysis on this design scheme of optimal-fuzzy-control structure was proposed. All of these works viewed the fuzzy system by individual rules, i.e., from local concept. It is, however, difficult for researchers to provide a theoretical demonstration on that the designed controller can reach global minimum effect, if the design scheme is based on local concept approach.

Technical contributions of this paper can be described as follows. The entire fuzzy system representation is proposed to 
maturate the formulation and simplification of the quadratic optimal fuzzy control problem. This global-concept approach might initiate and activate the research in global optimal fuzzy controller design. Further, a tricky unification of individual matrices into synthetical matrices generates a linear-like global system representation of a fuzzy system. This linear-like representation motivates us to develop the design scheme of a global optimal fuzzy controller in the way of general linear quadratic (LQ) approach. Moreover, a multistage-decomposition approach is adopted to transform the optimal control problem into an on-going stage-by-stage dynamic issue. This decomposition operation can speed up numerical solution, and keep the global optimality at the same time. The design scheme meets the necessary and sufficient condition of global optimum. The derived continuous fuzzy control law is theoretically demonstrated to be the best for the entire system to reach the optimal performance index. Finally, the in-depth analysis (controllability, observability, stability, degree of stability, and gain margin) in Section IV gives the complete perspective of all facets of the resultant closed-loop fuzzy system; we elicit that this kind of fuzzy controller can stabilize a continuous fuzzy system to any prescribed degree of stability; the corresponding closed-loop fuzzy system possesses an infinite gain margin; and the total energy of the system output of the closed-loop fuzzy system is absolutely finite. The design methodology is illustrated by one example.

\section{QUAdRATIC FuZzy PROBLem Formulation}

In this section, we shall propose an entire fuzzy system representation to maturate the formulation of the quadratic optimal fuzzy control problem. A sound unification of the individual matrices into synthetical matrices to form a linear-like global system representation of a fuzzy system helps the derivation of a theoretical design scheme of the quadratic optimal fuzzy controller. We consider a given nonlinear plant described by the so-called T-S type fuzzy model

$$
\begin{gathered}
R^{i}: \text { If } x_{1} \text { is } T_{1 i}, \ldots, x_{n} \text { is } T_{n i}, \text { then } \\
\dot{X}(t)=A_{i}(t) X(t)+B_{i}(t) u(t) \\
Y(t)=C(t) X(t), \quad i=1, \ldots, r
\end{gathered}
$$

where

$$
\begin{aligned}
& R^{i} \\
& x_{1}, \ldots, x_{n} \\
& T_{1 i}, \ldots, T_{n i} \\
& X(t)=\left[x_{1}, \ldots, x_{n}\right]^{t} \in \Re^{n} \\
& Y(t) \in \Re^{\prime} \\
& u(t) \in \Re^{m} \\
& A_{i}(t), B_{i}(t) \text { and } C(t)
\end{aligned}
$$

We then assume the desired controller is a rule-based nonlinear fuzzy controller

$$
\begin{aligned}
& R^{i}: \text { If } y_{1} \text { is } S_{1 i}, \ldots, y_{n^{\prime}} \text { is } S_{n^{\prime} i}, \text { then } \\
& u(t)=r_{i}(t), \quad i=1, \ldots, \delta
\end{aligned}
$$

where

$$
\begin{array}{ll}
y_{1}, \ldots, y_{n^{\prime}} & \text { elements of output vector } Y(t) ; \\
S_{1 i}, \ldots, S_{n^{\prime} i} & \text { input fuzzy terms in the } i \text { th control rule; } \\
u(t) \text { or } r_{i}(t) \in \Re^{m} & \text { plant input (i.e., control output) vector. }
\end{array}
$$

Then, a quadratic optimal fuzzy control problem is described as follows.

Problem 1: Given the rule-based fuzzy system in (1) with $X\left(t_{0}\right)=X_{0} \in \Re^{n}$ and a rule-based nonlinear fuzzy controller in (2), $t \in\left[t_{0}, t_{1}\right]$, find a controller $u^{*}(\cdot)$, which can minimize the quadratic cost functional

$$
\begin{aligned}
J(u(\cdot))= & \int_{t_{0}}^{t_{1}}\left[X^{t}(t) L(t) X(t)+u^{t}(t) u(t)\right] d t \\
& +X^{t}\left(t_{1}\right) Q X\left(t_{1}\right)
\end{aligned}
$$

over all possible inputs $u(\cdot)$ of class PC.

The grounding on distributed fuzzy subsystems and rule-based fuzzy controller brings the researchers in struggle to find out the controller $u^{*}(\cdot)$, which can achieve global minimum effect under quadratic performance consideration defined on the entire fuzzy system and fuzzy controller. In other words, it is a big troublesome challenge to achieve global optimal solution under local model consideration and, thus far, this issue has not been attacked directly even that the T-S type fuzzy model has been available for many years. Wang [10] tried to open the deadlock by considering a linear system (instead of fuzzy system) combined with a fuzzy controller. Tanaka et al. [16], [17] developed the LMI-based optimal fuzzy control by assuming a local linear-feedback gain-control structure. However, the quadratic optimal fuzzy control issue, in fact, remains fully open.

Since each penalty term in the performance index is with regard to the entire fuzzy system and controller, it flashes into our mind to formulate the distributed fuzzy subsystems and rulebased fuzzy controller into one equation from the global concept. Therefore, we "fuzzily blend" the well-known T-S type fuzzy model to obtain the entire fuzzy system formulation

$$
\begin{aligned}
\dot{X}(t) & =\sum_{i=1}^{r} h_{i}(X(t)) A_{i}(t) X(t)+\sum_{i=1}^{r} h_{i}(X(t)) B_{i}(t) u(t) \\
Y(t) & =\sum_{i=1}^{r} h_{i}(X(t)) C(t) X(t)
\end{aligned}
$$

and the entire fuzzy controller is

$$
u(t)=\sum_{i=1}^{\delta} w_{i}(Y(t)) r_{i}(t)
$$
and $n^{\prime} \times n$ matrices whose elements are known to be piecewise continuous (PC) and real-valued functions defined on positive real space $\Re_{+}$. with $\sum_{i=1}^{r} h_{i}(X(t))=1$ and $\sum_{i=1}^{\delta} w_{i}(Y(t))=1$, where $h_{i}(X(t))$ and $w_{i}(Y(t))$ denote, respectively, the normalized firing-strength of the $i$ th rule of the continuous fuzzy model and that of the $i$ th fuzzy control rule, i.e., 
$h_{i}(X(t))=\alpha_{i}(X(t)) / \sum_{i=1}^{r} \alpha_{i}(X(t))$ and $w_{i}(Y(t))=$ $\beta_{i}(Y(t)) / \sum_{i=1}^{\delta} \beta_{i}(Y(t))$ with $\alpha_{i}(X(t))=\prod_{j=1}^{n} \mu_{T_{j i}}\left(x_{j}(t)\right)$ and $\beta_{i}(Y(t))=\prod_{j=1}^{n^{\prime}} \mu_{S_{j i}}\left(y_{j}(t)\right)$, where $\mu_{T_{j i}}(X(t))$ and $\mu_{S_{j i}}\left(y_{j}(t)\right)$ are the membership functions of fuzzy terms $T_{j i}$ and $S_{j i}$, respectively. Thus, we obtain the formulation of the quadratic optimal fuzzy control problem in Problem 1 as follows.

Problem 2: Given the entire fuzzy system in (4) with the fuzzy controller $u(t)$ in (5) and $X\left(t_{0}\right)=X_{0} \in \Re^{n}, t \in\left[t_{0}, t_{1}\right]$, find the optimal control law $r_{i}^{*}(\cdot), i=1, \ldots, \delta$ to minimize the quadratic cost functional

$$
\begin{aligned}
J\left(r_{i}(\cdot)\right)= & \int_{t_{0}}^{t_{1}}\left[X^{t}(t) L(t) X(t)\right. \\
& \left.+\sum_{i=1}^{\delta} \sum_{j=1}^{\delta} w_{i}(Y(t)) w_{j}(Y(t)) r_{i}^{t}(t) r_{j}(t)\right] d t \\
& +X^{t}\left(t_{1}\right) Q X\left(t_{1}\right) .
\end{aligned}
$$

This kind of quadratic optimal control problems is, obviously, still too tough for us to engage in. Introducing the following synthetical matrices, $H(X(t)), W(Y(t)), A(t), B(t)$, and $R(t)$ can overcome the predicament, where

$$
\begin{aligned}
H(X(t)) & =\left[\begin{array}{lll}
h_{1}(X(t)) I_{n} & \ldots & h_{r}(X(t)) I_{n}
\end{array}\right] \\
W(Y(t)) & =\left[\begin{array}{lll}
w_{1}(Y(t)) I_{m} & \ldots & w_{\delta}(Y(t)) I_{m}
\end{array}\right] \\
A(t) & =\left[\begin{array}{c}
A_{1}(t) \\
\vdots \\
A_{r}(t)
\end{array}\right], \quad B(t)=\left[\begin{array}{c}
B_{1}(t) \\
\vdots \\
B_{r}(t)
\end{array}\right] \\
R(t) & =\left[\begin{array}{c}
r_{1}(t) \\
\vdots \\
r_{\delta}(t)
\end{array}\right]
\end{aligned}
$$

with $I_{n}$ and $I_{m}$ denoting the identity matrices of dimension $n$ and $m$, respectively. In other words, based on these synthetical notations, Problem 2 can be rewritten as the following final formulation:

Problem 3: Given a nonlinear but linear-like closed-loop fuzzy system

$$
\begin{aligned}
\dot{X}(t) & =H(X(t)) A(t) X(t)+H(X(t)) B(t) W(Y(t)) R(t) \\
Y(t) & =C(t) X(t)
\end{aligned}
$$

with $X\left(t_{0}\right)=X_{0} \in \Re^{n}$, find the optimal synthetical control law, $R^{*}(\cdot)$, to minimize the quadratic cost functional

$$
\begin{aligned}
J(R(\cdot))= & \int_{t_{0}}^{t_{1}}\left[X^{t}(t) L(t) X(t)\right. \\
& \left.+R^{t}(t) W^{t}(Y(t)) W(Y(t)) R(t)\right] d t \\
& +X^{t}\left(t_{1}\right) Q X\left(t_{1}\right) .
\end{aligned}
$$

This linear-like synthetical matrix representation for the entire $\mathrm{T}-\mathrm{S}$ type fuzzy system materializes the design of the global op- timal fuzzy controller in the way of general LQ approach, i.e., calculus-of-variation method.

In order to clarify the credit of the work, we now point out the kernel of the proposed global-concept approach as follows.

1) The entire fuzzy system representation, obtained by fuzzily merging the distributed rule-based T-S-type fuzzy subsystems into one, is proposed to maturate the formulation and simplification of the quadratic optimal fuzzy control problem. This global-concept approach might initiate and activate the research in global optimal fuzzy controller design.

2) The individual matrices $\left(A_{i}(k)\right.$ and $\left.B_{i}(k), i=1, \ldots, r\right)$ and normalized membership functions $\left(h_{i}(X(k))\right.$, $i=1, \ldots, r$, and $\left.w_{i}(Y(k)), i=1, \ldots, \delta\right)$ are unified into synthetical matrices $(A(k), B(k), H(X(k))$, and $W(Y(k)))$. This unification generates a linear-like global system representation of a fuzzy system with the value of each element of the nonlinear terms $(H(X(k))$ and $W(Y(k))$ ) being located in segment [0,1]. This linear-like representation motivates us to develop the design scheme of global optimal fuzzy controller in the way of general LQ approach, i.e., calculus-of-variation method.

3) A multistage-decomposition approach (Lemma 1) is adopted to transform the optimal control problem into an on-going stage-by-stage dynamic issue. Therefore, the optimal solutions can be resolved from $N$ segmental nonlinear two-point boundary-value problem (TPBVP) instead of the nonlinear TPBVP for the entire horizon. This decomposition operation can speed up numerical solution and keep the global optimality at the same time.

4) The existence of $\bar{N}$, denoting the number of stages at which membership functions can be assumed to be invariant during the whole single stage, is assumed to make the backward recursive Riccati-like equation available. This avoids the high computational complexity of the collocation method at the expense of approximate optimality due to the time-invariant assumption. A procedure including a dynamical decomposition algorithm is proposed to justify the time-invariant assumption in practice.

5) For time-invariant case, the algebraic Riccati-like equation is available in the aid of Lemma 2 and, thus, the finitehorizon optimal solution is coincident with the asymptotic (infinite-horizon) optimal solution.

6) The in-depth analysis (stability, degree of stability, and gain margin) in Section IV gives the complete perspective of all facets of the resultant closed-loop fuzzy system.

It is important for us to mention here that the process of integrating all distributed fuzzy subsystems into one equation to describe the entire fuzzy system is necessary in order to find out the global optimal solution. The proposed fuzzily blended entire fuzzy system in (4) provides a practical way to work out the global optimal solution. However, even each fuzzy subsystem in T-S model is linear, the fuzzily blended entire fuzzy system in (4) is complicated and highly nonlinear. The further proposed synthetical matrix representation of the entire fuzzy system in (7) shall, in the sense of global optimality, lower down the order 
and difficulty of the problem. This kind of global system representation will be the foundation and kernel of the following fuzzy controller design scheme.

\section{OPTIMAL FUZZY CONTROLLER DESIGN}

We are going to design the optimal fuzzy controllers for continuous fuzzy system with finite-horizon in Section III-A and for that with infinite-horizon in Section III-B.

\section{A. Finite-Horizon Problem}

By describing the fuzzy system from the global concept in Section II, our quadratic optimal fuzzy control problem for the T-S type fuzzy system can be formulated and simplified into Problem 3 in Section II. Since the membership functions in the fuzzy controller and fuzzy system are piecewise continuous, it is reasonable to make the following assumption around the extreme points.

Assumption 1: The membership functions of slightly perturbed extremes are almost equivalent to those of the extremes, i.e., $\mu_{T_{j i}}\left(x_{j}^{*}(t)\right)=\mu_{T_{j i}}\left(x_{j}^{*}(t)+\epsilon_{1} z_{j}(t)\right), j=1, \ldots, n$, $i=1, \ldots, r$, and $\mu_{S_{j i}}\left(y_{j}^{*}(t)\right)=\mu_{T_{j i}}\left(y_{j}^{*}(t)+\epsilon_{2} v_{j}(t)\right)$, $j=1, \ldots, n^{\prime}, i=1, \ldots, \delta$, where $\epsilon_{i}, i=1,2$ are very small positive value.

For frequently used membership functions such as bell-shaped, triangular, and trapezoid membership functions, this assumption soundly holds. Further, from the essence of the dynamic programming formalism, we make the following multistage decomposition of optimization scheme.

Lemma 1 (Multistage Decomposition): A foregoing optimization scheme is a dynamic allocation process or a successive multistage decision process. In other words, if we let $t_{0}=t_{0}^{1}, t_{1}=t_{1}^{N}, t_{0}^{i}=t_{1}^{i-1}, i=2, \ldots, N ; \Delta t^{i}=t_{1}^{i}-t_{0}^{i}, i=$ $1, \ldots, N$ and define (see equation at the bottom of the page) with regard to the state resulting from the previous decision, i.e., $X\left(t_{0}^{1}\right)=X_{0} ; X\left(t_{0}^{i}\right)=X^{*}\left(t_{1}^{i-1}\right), i=2, \ldots, N$, then

$$
\phi(X(\cdot), u(\cdot))=\Phi^{1}(X(\cdot), u(\cdot))+\cdots+\Phi^{N}(X(\cdot), u(\cdot)) .
$$

Proof: See Appendix.

We shall first decompose the optimization problem into an on-going stage-by-stage dynamic issue with the aid of Lemma 1. Then, successively focusing on only one stage at a time, we use the calculus of variations method combined with Lagrange multiplier method to obtain the necessary and sufficient condition for global optimum.

Theorem 1: For the fuzzy system in (1) and fuzzy controller in (2) with nonsharp-profile membership functions, let $\left(X^{*}(t), R^{*}(t)\right), t \in\left[t_{0}, t_{1}\right]$ be the optimal solution with respect to $J(R(\cdot))$ in (8) and $\left(X^{i^{*}}(t), R^{i^{*}}(t)\right), t \in\left[t_{0}^{i}, t_{1}^{i}\right]$ be the $i$ th-stage optimal solution with respect to

$$
\begin{aligned}
J^{i}(R(\cdot))= & \int_{t_{0}^{i}}^{t_{1}^{i}}\left(X^{t}(t) L(t) X(t)\right. \\
& \left.+R^{t}(t) W^{t}(Y(t)) W(Y(t)) R(t)\right) \\
& +X^{t}\left(t_{1}^{i}\right) Q^{i} X\left(t_{1}^{i}\right)
\end{aligned}
$$

where $i=1, \ldots, N ; Q^{i}$ equals to $Q$ at the $N$ th stage and is a zero matrix, otherwise; the initial condition $X\left(t_{0}^{1}\right)=X_{0}$ and $X\left(t_{0}^{i}\right)=X^{*}\left(t_{1}^{i-1}\right)$ for $i=2, \ldots, N$. Then

1) $\left(X^{*}(t), R^{*}(t)\right)=\left(X^{i^{*}}(t), R^{i^{*}}(t)\right)$ for all $t \in\left[t_{0}^{i}, t_{1}^{i}\right]$, $i=1, \ldots, N ;$ and $t_{0}^{1}=t_{0}, t_{1}^{N}=t_{1}, t_{0}^{i}=t_{1}^{i-1}, i=$ $2, \ldots, N$

2) for the $i$ th stage $t \in\left[t_{0}^{i}, t_{1}^{i}\right]$ the optimal synthetical control law is

$$
\begin{aligned}
R^{*}(t)= & -W^{t}\left(Y^{*}(t)\right)\left[W\left(Y^{*}(t)\right) W^{t}\left(Y^{*}(t)\right)\right]^{-1} \\
& \times B^{t}(t) H^{t}\left(X^{*}(t)\right) P(t)
\end{aligned}
$$

and the corresponding global minimizer is

$$
u^{*}(t)=-B^{t}(t) H^{t}\left(X^{*}(t)\right) P(t)
$$

where $P(t)$ satisfies the following TPBVP

$$
\begin{aligned}
\frac{d}{d t} & {\left[\begin{array}{c}
X^{*}(t) \\
P(t)
\end{array}\right] } \\
& =\left[\begin{array}{cc}
H\left(X^{*}(t)\right) A(t) & -H\left(X^{*}(t)\right) B(t) B^{t}(t) H^{t}\left(X^{*}(t)\right) \\
-L(t) & -A^{t}(t) H^{t}\left(X^{*}(t)\right)
\end{array}\right] \\
& \times\left[\begin{array}{c}
X^{*}(t) \\
P(t)
\end{array}\right]
\end{aligned}
$$

with $P\left(t_{1}^{i}\right)=Q^{i} X^{*}\left(t_{1}^{i}\right)$, where $X^{*}(\cdot)$ is the corresponding optimal state trajectory.

Proof: See Appendix.

We should emphasize here the multistage-decomposition approach in Lemma 1 can transform the optimal control problem into an on-going stage-by-stage dynamic issue. Therefore, the optimal solutions can be resolved from $N$ segmental nonlinear TPBVP instead of the nonlinear TPBVP for the entire horizon. This decomposition operation can speed up numerical solution, and keep the global optimality at the same time.

Moreover, though the membership functions are dependent on the system state, the state-penalty term $X^{t}(t) L(t) X(t)$ in the cost functional in (3) or (8) can encourage a smooth optimal trajectory [19]. For a chosen nonsharp membership function

and

$$
\phi(X(\cdot), u(\cdot))=\min _{u_{\left[t_{0}, t_{1}\right]}} \int_{t_{0}}^{t_{1}}\left[X^{t}(t) L(t) X(t)+u^{t}(t) u(t)\right] d t+X^{t}\left(t_{1}\right) Q X\left(t_{1}\right)
$$

$$
\phi^{i}(X(\cdot), u(\cdot))= \begin{cases}\min _{u_{\left[t_{0}^{i}, t_{1}^{i}\right]}} \int_{t_{0}^{i}}^{t_{1}^{i}}\left[X^{t}(t) L(t) X(t)+u^{t}(t) u(t)\right] d t, & i=1, \ldots, N-1 \\ \min _{u_{\left[t_{0}^{N}, t_{1}\right]}} \int_{t_{0}^{N}}^{t_{1}}\left[X^{t}(t) L(t) X(t)+u^{t}(t) u(t)\right] d t+X^{t}\left(t_{1}\right) Q X\left(t_{1}\right), & i=N\end{cases}
$$


profile, it is, in concept, reasonable and workable to increase the sampling frequency such that the membership function of the optimal state $X^{*}(t)$ remains almost invariant during each stage. In other words, we can further adjust the division, i.e., enlarge $N$, to the extent that $H(X(t))$ and $W(Y(t))$ are almost invariant during the whole single stage, and use $H_{i}$ and $W_{i}$ to denote $H(X(t))$ and $W(Y(t))$, respectively, at the $i$ th stage. Then, the optimal synthetical control law becomes

$$
R^{*}(t)=-W_{i}^{t}\left[W_{i} W_{i}^{t}\right]^{-1} B^{t}(t) H_{i}^{t} P(t), \quad t \in\left[t_{0}^{i}, t_{1}^{i}\right]
$$

where $P(t)$ satisfies the following linear TPBVP

$$
\frac{d}{d t}\left[\begin{array}{c}
X^{*}(t) \\
P(t)
\end{array}\right]=\left[\begin{array}{cc}
H_{i} A(t) & -H_{i} B(t) B^{t}(t) H_{i}^{t} \\
-L(t) & -A^{t}(t) H_{i}^{t}
\end{array}\right]\left[\begin{array}{c}
X^{*}(t) \\
P(t)
\end{array}\right]
$$

with $H_{1}=H\left(X_{0}\right), W_{1}=W\left(Y\left(t_{0}\right)\right), X\left(t_{0}^{1}\right)=X_{0} ; H_{i}=$ $H\left(X^{*}\left(t_{1}^{i-1}\right)\right), W_{i}=W\left(Y^{*}\left(t_{1}^{i-1}\right)\right), X\left(t_{0}^{i}\right)=X^{*}\left(t_{1}^{i-1}\right), \forall i=$ $2, \ldots, N ; P\left(t_{1}^{i}\right)=Q^{i} X^{*}\left(t_{1}^{i}\right), \forall i=1, \ldots, N$. Furthermore, Let $P(t)=K(t) X^{*}(t)$. Then, (15) is equivalent to the following segmental Riccati-like differential equation (DE):

$$
\begin{aligned}
-\dot{K}(t)= & L(t)-K(t) H_{i} B(t) B^{t}(t) H_{i}^{t} K(t) \\
& +A^{t}(t) H_{i}^{t} K(t)+K(t) H_{i} A(t)
\end{aligned}
$$

where $t \in\left[t_{0}^{i}, t_{1}^{i}\right], i=1, \ldots, N ; t_{0}^{1}=t_{0}, t_{1}^{N}=t_{1} ; K\left(t_{1}^{i}\right)=$ $Q^{i}$

We further define $\bar{N}$ to be the number of stages at which membership functions can be assumed to be invariant during the whole single stage. Then, the backward Riccati-like DE in (16) becomes available due to the existence of $\bar{N}$. This avoids the high computational complexity of the collocation method at the expense of approximate optimality due to the time-invariant assumption. We can ensure this assumption by checking the following condition at the starting time-instant of the $i$ th stage, saying $t_{0}^{i}$

$$
\left\|\frac{d H\left(X^{*}(\tau)\right)}{d \tau}\right\|_{\tau=t_{0}^{i}} \leq \kappa_{H_{1}}
$$

and then keeping checking the following condition to find the proper length of this stage:

$$
\left\|H\left(X^{*}(t)\right)-H\left(X^{*}\left(t_{0}^{i}\right)\right)\right\| \leq \kappa_{H_{2}}, \quad \forall t \in\left[t_{0}^{i}, t_{1}^{i}\right]
$$

where $\kappa_{H_{1}}$ and $\kappa_{H_{2}}$ are the given tolerance to ensure the almost-invariant criteria. The first inequality in (17) ensures that the membership degrees corresponding to the optimal trajectory $X^{*}(\cdot)$ at time-instant $t_{0}^{i}$ does not change in abrupt shape and also gives a hint that an almost-invariant-membership-function stage from time-instant $t_{0}^{i}$ is achievable. The second inequality in (18) is to check the almost-invariant criteria for the entire $i$ th stage to find out the length (time period) of the stage and then can also provide the information about the value of $\bar{N}$. These two inequalities are used to check the time-invariant criteria in the dynamic decomposition algorithm (DDA) in Section V. Now, we summarize the previous derivation in the following assertion. $\square$

Theorem 2: For the fuzzy system and fuzzy controller represented, respectively, by (1) and (2), let $\left(X^{*}(t), R^{*}(t)\right), t \in$ $\left[t_{0}, t_{1}\right]$ be the optimal solution with respect to $J(R(\cdot))$ in $(8)$ and $\left(X^{i^{*}}(t), R^{i^{*}}(t)\right), t \in\left[t_{0}^{i}, t_{1}^{i}\right]$ be the $i$ th-stage optimal solution with respect to $J^{i}(R(\cdot))$ in (10). If $N>\bar{N}$ then

1) $\left(X^{*}(t), R^{*}(t)\right)=\left(X^{i^{*}}(t), R^{i^{*}}(t)\right)$ for all $t \in\left[t_{0}^{i}, t_{1}^{i}\right]$, $i=1, \ldots, N$; and $t_{0}^{1}=t_{0}, t_{1}^{N}=t_{1}, t_{0}^{i}=t_{1}^{i-1}, i=$ $2, \ldots, N$

2) for the $i$ th stage $t \in\left[t_{0}^{i}, t_{1}^{i}\right]$, the optimal synthetical control law is

$$
R^{i^{*}}(t)=-W_{i}^{t}\left[W_{i}^{t} W_{i}\right]^{-1} B^{t}(t) H_{i}^{t} \pi^{i}\left(t, t_{1}^{i}\right) X^{i^{*}}(t)
$$

and the corresponding global minimizer is

$$
u^{i^{*}}(t)=-B^{t}(t) H_{i}^{t} \pi^{i}\left(t, t_{1}^{i}\right) X^{i^{*}}(t)
$$

where $\pi^{i}\left(t, t_{1}^{i}\right)$ is the symmetric positive semidefinite solution of the segmental Riccati-like DE in (16); the $i$ th-stage optimal trajectory is

$$
\dot{X}^{i^{*}}(t)=\left[H_{i} A(t)-H_{i} B(t) B^{t}(t) H_{i}^{t} \pi^{i}\left(t, t_{1}^{i}\right)\right] X^{i^{*}}(t)
$$

3) the minimum performance index is equal to $\sum_{i=1}^{N} X^{i^{*}}\left(t_{0}^{i}\right) \pi^{i}\left(t_{0}^{i}, t_{1}^{i}\right) X^{i^{*}}\left(t_{0}^{i}\right)$.

Proof: This theorem follows the above inference.

So far, we have solved the optimal fuzzy control problem by finding the optimal solution to the general time-varying case. In the classical LQ optimal control problem, a time-invariant system will give rise to time-invariant linear optimal control law. We are now eager to know if this phenomenon exists in each segmental fuzzy system. Some useful lemmas are demonstrated below in order to develop the design scheme of optimal fuzzy control law regarding to the time-invariant fuzzy system.

Lemma 2: Consider a dynamical system, $\dot{X}(t)=$ $f(X(t), u(t), t)$, with $X\left(t_{0}^{i}\right)=X_{0}^{i}$. Let the pair $\left(X^{*}(\cdot), u^{*}(\cdot)\right)$ be the infinite-horizon optimal solution with the performance index $J(u(\cdot))=\int_{t_{0}^{i}}^{\infty} f_{0}(X(t), u(t), t) d t$ and the pair $\left(\bar{X}^{*}(\cdot), \bar{u}^{*}(\cdot)\right)$ be the finite-horizon optimal solution with respect to $\bar{J}(u(\cdot))=\int_{t_{0}^{i}}^{t_{1}^{i}} f_{0}(X(t), u(t), t) d t$, where $f(\cdot, \cdot, \cdot)$, $f_{0}(\cdot, \cdot, \cdot) \in \mathrm{PC}\left(\Re^{n}, \Re^{m}, \Re\right)$, a mapping from $\Re^{n} \times \Re^{m} \times \Re$ to real-valued functions of class PC. If $X\left(t_{1}^{i}\right)$ is a free point, then $\left(\bar{X}^{*}(t), \bar{u}^{*}(t)\right)=\left(X^{*}(t), u^{*}(t)\right)$ for all $t \in\left[t_{0}^{i}, t_{1}^{i}\right]$.

Proof: Assume the infinite-horizon optimal solution for some $t \in\left[t_{0}^{i}, t_{1}^{i}\right]$ is not the finite-horizon optimal solution, then

$$
\int_{t_{0}^{i}}^{t_{1}^{i}} f_{0}\left(\bar{X}^{*}(t), \bar{u}^{*}(t), t\right) d t<\int_{t_{0}^{i}}^{t_{1}^{i}} f_{0}\left(X^{*}(t), u^{*}(t), t\right) d t
$$

If we define a decision sequence $\hat{u}(t)$ as

$$
(\hat{X}(t), \hat{u}(t))= \begin{cases}\left(\bar{X}^{*}(t) \bar{u}^{*}(t)\right), & t \in\left[t_{0}^{i}, t_{1}^{i}\right] \\ \left(X^{*}(t), u^{*}(t)\right), & t \in\left[t_{1}^{i}, \infty\right)\end{cases}
$$

where $\hat{X}(t)$ denotes the corresponding state trajectory, then

$$
\begin{aligned}
\int_{t_{0}^{i}}^{\infty} f_{0}(\hat{X}(t), \hat{u}(t), t) d t= & \int_{t_{0}^{i}}^{t_{1}^{i}} f_{0}\left(\bar{X}^{*}(t), \bar{u}^{*}(t), t\right) d t \\
& +\int_{t_{1}^{i}}^{\infty} f_{0}\left(X^{*}(t), u^{*}(t), t\right) d t \\
& <\int_{t_{0}^{i}}^{\infty} f_{0}\left(X^{*}(t), u^{*}(t), t\right) d t
\end{aligned}
$$


This conflicts with that $\left(X^{*}(t), u^{*}(t)\right)$ is the infinite-horizon optimal solution. Thus, $\left(\bar{X}^{*}(t), \bar{u}^{*}(t)\right)=\left(X^{*}(t), u^{*}(t)\right)$ holds for all $t \in\left[t_{0}^{i}, t_{1}^{i}\right]$ positively.

Furthermore, we develop two lemmas below to link the controllability and observability of the fuzzy subsystem to those of the entire fuzzy system.

Lemma 3 (Controllability): $\left(A_{i}, B_{i}\right)$ is completely controllable (CC) for all $i=1, \ldots, r$ if and only if $\operatorname{rank}\left[\lambda I_{n}-H(X(k)) A H(X(k)) B\right]=n$, for all $X(t) \in \Re^{n}$ and $\lambda \in \mathcal{C}$.

Proof: See Appendix.

Lemma 4 (Observability): $\left(A_{i}, C\right)$ is completely observable (CO) for all $i=1, \ldots, r$, if and only if

$$
\operatorname{rank}\left[\begin{array}{c}
\lambda I_{n}-H(X(t)) A \\
C
\end{array}\right]=n, \quad \forall X(t) \in \Re^{n}, \quad \lambda \in \mathcal{C} .
$$

Proof: The proof is similar to that in Lemma 3.

Now, the aforementioned inference can be summarized into the following lemma.

Lemma 5: For each segmental dynamical fuzzy system

$$
\begin{aligned}
\dot{X}(t) & =H_{i} A X(t)+H_{i} B W_{i} R(t), \\
Y(t) & =C X(t)
\end{aligned}
$$

with $X\left(t_{0}^{i}\right)$ known. If $\left(A_{i}, B_{i}\right)$ is $\mathrm{CC}$ and $\left(A_{i}, C\right)$ is $\mathrm{CO}$ for all $i=1, \ldots, r$, then

1) there exists an unique $n \times n$ symmetric positive semidefinite solution $\pi_{\infty}^{i}$ of the steady state Riccati equation (SSRE)

$$
A^{t} H_{i}^{t} K+K H_{i} A-K H_{i} B B^{t} H_{i}^{t} K+C^{t} C=0
$$

2) the asymptotically optimal control law is

$$
R_{\infty}^{i^{*}}(t)=-W_{i}^{t}\left[W_{i} W_{i}^{t}\right]^{-1} B^{t} H_{i}^{t} \pi_{\infty}^{i} X_{\infty}^{i^{*}}(t), \quad t \in\left[t_{0}^{i}, \infty\right)
$$

which minimizes

$$
J_{\infty}^{i}(R(\cdot))=\int_{t_{0}^{i}}^{\infty}\left[X^{t}(t) L X(t)+R^{t}(t) W_{i}^{t} W_{i} R(t)\right] d t
$$

3) the optimal closed-loop fuzzy system

$$
\dot{X}_{\infty}^{i^{*}}(t)=\left(H_{i} A-H_{i} B B^{t} H_{i}^{t} \pi_{\infty}^{i}\right) X_{\infty}^{i^{*}}(t), \quad t \in\left[t_{0}^{i}, \infty\right)
$$

is asymptotically and exponentially stable.

Proof: We know, from Lemmas 3 and 4, $\left(A_{i}, B_{i}\right)$ is $\mathrm{CC}, \forall i=1, \ldots, r$, if and only if $\operatorname{rank}\left[\lambda I_{n}-\right.$ $H(X(t)) A H(X(t)) B]=n, \forall X(t) \in \Re^{n}, \forall \lambda \in \mathcal{C}$ and, accordingly, $\operatorname{rank}\left[\lambda I_{n}-H_{i} A H_{i} B\right]=n, \forall \lambda \in \sigma\left(H_{i} A\right)$, $i=1, \ldots, N$. Also, $\left(A_{i}, C\right)$ is CO, $\forall i=1, \ldots, r$, if and only if $\operatorname{rank}\left[\begin{array}{c}\lambda I_{n}-H(X(t)) A \\ C\end{array}\right]=n, \forall X \in \Re^{n}, \forall \lambda \in \mathcal{C}$, which ensures $\operatorname{rank}\left[\begin{array}{c}\lambda I_{n}-H_{i} A \\ C\end{array}\right]=n, \forall \lambda \in \mathcal{C}$. Therefore, $\left(A_{i}, B_{i}\right)$ $\mathrm{CC}$ and $\left(A_{i}, C\right) \mathrm{CO}, \forall i=1, \ldots, r$, guarantee $\left(H_{i} A, H_{i} B\right) \mathrm{CC}$ and $\left(H_{i} A, C\right) \mathrm{CO}$, respectively. Then, by the classical linear quadratic optimal control theorem [20], we have the optimal solution for the segmental fuzzy system in (22).

A more implementable theorem for the time-invariant fuzzy subsystems can be extracted based on the aforementioned lemmas and Theorem 2, which considers the time-varying fuzzy subsystems.

Theorem 3 (Time-Invariant): Consider the time-invariant fuzzy system and fuzzy controller described, respectively, by (1) and (2) with $L=C^{t} C$ in (8). Let $\left(X^{*}(t), R^{*}(t)\right.$ ), $t \in\left[t_{0}, t_{1}\right]$, denote the optimal solution with respect to $J(R(\cdot))$ in $(8),\left(X^{i^{*}}(t), R^{i^{*}}(t)\right), t \in\left[t_{0}^{i}, t_{1}^{i}\right]$, denote the $i$ th-stage optimal solution with respect to $J^{i}(R(\cdot))$ in (10) and $\left(X_{\infty}^{i^{*}}(t), R_{\infty}^{i^{*}}(t)\right), t \in\left[t_{0}^{i}, t_{1}^{i}\right]$, be the $i$ th-stage asymptotically optimal solution with respect to $J_{\infty}^{i}(R(\cdot))$ in (25). If $N>\bar{N}$, $\left(A_{i}, B_{i}\right)$ is $\mathrm{CC}$ and $\left(A_{i}, C\right)$ is $\mathrm{CO}$, for all $i=1, \ldots, r$, then

1) see (27) at the bottom of the page where $t_{0}^{i}=t_{1}^{i-1}, i=$ $2, \ldots, N ; t_{0}^{1}=t_{0}$

2) for the $i$ th stage, $i=1, \ldots, N-1$, the optimal synthetical control law is $R_{\infty}^{i^{*}}(\cdot)$ in (24), and the optimal trajectory is $X_{\infty}^{i^{*}}(\cdot)$ in (26), where $\pi_{\infty}^{i}$ is the unique symmetric positive semidefinite solution of the SSRE in (23);

3 ) as for the last stage, the $N$ th stage, the optimal synthetical control law is $R^{i^{*}}(\cdot)$ in (19) and the optimal trajectory is $X^{i^{*}}(\cdot)$ in (21), where $\pi^{i}\left(t, t_{1}^{i}\right)$ is the symmetric positive semidefinite solution of the segmental Riccati DE in (16);

4) the minimum performance index is

$$
\begin{aligned}
\min _{R_{\left[t_{0}, t_{1}\right]}} J(R(\cdot))= & \sum_{i=1}^{N-1}\left[X_{\infty}^{i^{*^{t}}}\left(t_{0}^{i}\right) \pi_{\infty}^{i} X_{\infty}^{i^{*}}\left(t_{0}^{i}\right)\right] \\
& +X^{N^{*^{t}}}\left(t_{0}^{N}\right) \pi^{N}\left(t_{0}^{N}, t_{1}\right) X^{N^{*}}\left(t_{0}^{N}\right) .
\end{aligned}
$$

Proof:

2) Based on Lemma 1, the whole optimization is decomposed into an $N$-stage decision process with, at each stage, the initial state resulting from the decision of its previous stage. Now, our optimal fuzzy control problem-Problem 1-can be attacked in the following two issues, with both regarding to the same dynamical fuzzy system described by (7) except that the initial stage is $X\left(t_{0}^{i}\right)$ and the time interval is $\left[t_{0}^{i}, t_{1}^{i}\right]$ for the $i$ th stage

$$
\text { (a) } \begin{aligned}
\min _{R_{\left[t_{0}^{N}, t_{1}\right]}} \int_{t_{0}^{N}}^{t_{1}}\left[X^{t}(t) L(t) X(t)\right. \\
\left.\quad+R^{t}(t) W^{t}(Y(t)) W(Y(t)) R(t)\right] d t \\
\quad+X^{t}\left(t_{1}\right) Q X\left(t_{1}\right)
\end{aligned}
$$

$$
\left(X^{*}(t), R^{*}(t)\right)= \begin{cases}\left(X_{\infty}^{i^{*}}(t), R_{\infty}^{i^{*}}(t)\right), & \forall t \in\left[t_{0}^{i}, t_{1}^{i}\right], \quad i=1, \ldots, N-1 \\ \left(X^{i^{N}}(t), R^{i^{N}}(t)\right), & \forall t \in\left[t_{0}^{N}, t_{1}\right]\end{cases}
$$




$$
\text { (b) } \begin{aligned}
\min _{R_{\left[t_{0}^{i}, t_{1}^{i}\right]}} \int_{t_{0}^{i}}^{t_{1}^{i}}\left[X^{t}(t) L(t) X(t)\right. \\
\left.+R^{t}(t) W^{t}(Y(t)) W(Y(t)) R(t)\right] d t \\
\quad i=1, \ldots, N-1 .
\end{aligned}
$$

Furthermore, by Lemma 2, the optimal solution with respect to (29) can be regarded as the one with respect to

$$
\begin{aligned}
& \min _{R_{\left[t_{0}^{i}, \infty\right]}} \int_{t_{0}^{i}}^{\infty}\left[X^{t}(t) L(t) X(t)\right. \\
& \left.+R^{t}(t) W^{t}(Y(t)) W(Y(t)) R(t)\right] d t \\
& \quad i=1, \ldots, N-1 .
\end{aligned}
$$

Notice that this equivalence only exists on the time in period $\left[t_{0}^{i}, t_{1}^{i}\right]$. Therefore, hereinafter, we can pay attention only to (30) for the time interval $\left[t_{0}^{i}, t_{1}^{i}\right], i=$ $1, \ldots, N-1$ and to (28) for the time interval $\left[t_{0}^{N}, t_{1}\right]$.

3) For $N>\bar{N}, H(X(t))$ and $W(Y(t))$ in the dynamic fuzzy system described by (7) can be replaced, respectively, by constant matrices $H_{i}$ and $W_{i}$ for the $i$ th stage. Therefore, the whole fuzzy system in (7) can be rewritten as a linear system represented by (22). The $N$ th-stage optimal solution, indeed, follows from the optimal solution in Theorem 2. As for the other stages, we know from the proof of Lemmas $5,\left(A_{i}, B_{i}\right) \mathrm{CC}$ and $\left(A_{i}, C\right) \mathrm{CO}$, $\forall i=1, \ldots, r$ guarantee, respectively, $\left(H_{i} A, H_{i} B\right) \mathrm{CC}$ and $\left(H_{i} A, C\right) \mathrm{CO}, \forall i=1, \ldots, N$, where $r$ and $N$ are, respectively, the number of rules of the fuzzy system in (1) and the number of stages of the process described by the dynamical fuzzy system in (22). Hence, we can obtain the optimal solution for the first $N-1$ stages via Lemma 5.

Hence, for the first $N-1$ stages, a time-invariant fuzzy system can still give rise to the time-invariant linear optimal fuzzy control law.

At the moment, we extend our study in designing the optimal fuzzy controller from the free-end point problem to the fixed-end point problem in which a desired final state (the target) is given. The fixed end-point quadratic optimal fuzzy control problem is defined as follows.

Problem 4: Given the fuzzy system in (7) with $X\left(t_{0}\right)=X_{0}$, $X\left(t_{1}\right)=X_{1} \in \Re^{n}$ and $t \in\left[t_{0}, t_{1}\right]$, find $R^{*}(\cdot)$ to minimize $J(R(\cdot))$ in $(8)$.

The procedure to solve this problem is similar to that for solving Problem 1. Therefore, we only summarize the generalized theorem as follows.

Theorem 4: For the fuzzy system in (1) with $X\left(t_{1}\right)=X_{1}$ and the fuzzy controller in (2), the optimal control law with respect to $J(R(\cdot))$ in (8) is $R^{*}(t)$ in (11) and the corresponding global minimizer is $u^{*}(t)$ in (12), where $P(t)$ and optimal trajectory $X^{*}(\cdot)$ satisfy TPBVP in (13) with $X\left(t_{0}\right)=X_{0}$ and $X\left(t_{1}\right)=X_{1}$.

Proof: The proof is similar to that in Theorem 1.

Since the final state is constrained, Problem 4 does not belong to the standard foregoing optimization problem and, accordingly, the successive multistage decomposition approach is now unavailable for this case.

\section{B. Infinite-Horizon Problem}

The purpose of this section is to design the optimal fuzzy controller concerning the infinite-horizon problem, which is the case that the operating time goes to infinity or is much larger than the time-constant of the dynamic system. It is critical to notice the problem: Does the minimal performance index finitely exists? We introduce the concept proposed by Machi and Strauss [21]: If the linearized system of a nonlinear system with respect to (w.r.t) some state $X_{o} \in \Re^{n}$ is $C C$, then $X_{o}$ is an interior point of the controllable set (the set of all initial points which can be steered to the target). Now, the linearized system of the fuzzy system in (7) with respect to point $X_{O}$ is

$$
\dot{X}(t)=H\left(X_{o}\right) A(t) X(t)+H\left(X_{o}\right) B(t) u(t) .
$$

Therefore, to ensure that our problem is solvable, it is necessary that the pair $\left(H\left(X_{o}\right) A(\cdot), H\left(X_{o}\right) B(\cdot)\right)$ is controllable at all time and for all $X_{o} \in \Re^{n}$. We can now find out the design scheme of the infinite-horizon optimal fuzzy controller.

Theorem 5: For the fuzzy system and fuzzy controller in (1) and (2), respectively, if the linearized fuzzy system in (31) is controllable and there exists on $\left[t_{0}, \infty\right)$ an $n \times n$ symmetric positive semidefinite solution $\phi\left(t, t_{0}\right)$ to the forward Riccati-like DE

$$
\begin{aligned}
\dot{K}(t)= & L(t)-K(t) H(X(t)) B(t) B^{t}(t) H^{t}(X(t)) K(t) \\
& -A^{t}(t) H^{t}(X(t)) K(t)-K(t) H(X(t)) A(t)
\end{aligned}
$$

where $\dot{K} \geq 0$ and the initial value of the dependent variable $K\left(t_{0}\right)=0$, then there exists a optimal synthetical control law

$$
\begin{aligned}
R^{*}(t)= & W^{t}\left(Y^{*}(t)\right)\left[W\left(Y^{*}(t)\right) W^{t}\left(Y^{*}(t)\right)\right]^{-1} \\
& \times B^{t} H^{t}\left(X^{*}(t)\right) \phi\left(t, t_{0}\right) X^{*}(t)
\end{aligned}
$$

which minimizes

$$
\begin{aligned}
J(R(\cdot))= & \int_{t_{0}}^{\infty}\left[X^{t}(t) L(t) X(t)\right. \\
& \left.+R^{t}(t) W^{t}(Y(t)) W(Y(t)) R(t)\right] d t
\end{aligned}
$$

and the corresponding global minimizer is

$$
u^{*}(t)=B^{t} H^{t}\left(X^{*}(t)\right) \phi\left(t, t_{0}\right) X^{*}(t) .
$$

The dynamics of the resultant closed-loop fuzzy system is described by

$$
\begin{aligned}
\dot{X}^{*}(t)= & {\left[H\left(X^{*}(t)\right) A(t)\right.} \\
& \left.-H\left(X^{*}(t)\right) B(t) B^{t}(t) H^{t}\left(X^{*}(t)\right) \phi\left(t, t_{0}\right)\right] X^{*}(t) \\
& t \in\left[t_{0}, \infty\right]
\end{aligned}
$$

with $X\left(t_{0}\right)=X_{0}$.

Proof: See Appendix.

Though the firing-strength matrix $H(X(\cdot))$ is state dependent, Theorem 5 is actually implementable since the Riccati-like $\mathrm{DE}$ in (32) is of forward, just as the state equation in (7). However, it is indeed complicated in computational aspect. Therefore, a multistage approach as in Section III-A is still preferable.

Theorem 6: For the fuzzy system and fuzzy controller described by (1) and (2), respectively, let $\left(X_{\infty}^{*}(t), R_{\infty}^{*}(t)\right), t \in$ $\left[t_{0}, \infty\right)$, be the optimal solution with respect to $J(R(\cdot))$ in (34), 
and $\left(X^{i^{*}}(t), R^{i^{*}}(t)\right), t \in\left[t_{0}^{i}, t_{1}^{i}\right]$, be the $i$ th-stage optimal solution with respect to (37), shown at the bottom of the page. If $N>\bar{N}$ and the linearized fuzzy system in (31) is controllable, then

2) $\left(X_{\infty}^{*}(t), R_{\infty}^{*}(t)\right)=\left(X^{i^{*}}(t), R^{i^{*}}(t)\right), t \in\left[t_{0}^{i}, t_{1}^{i}\right], i=$ $1, \ldots, N$, where $t_{0}^{i}=t_{1}^{i-1}, i=2, \ldots, N ; t_{0}^{1}=t_{0}, t_{1}^{N}=$ $\infty$

3) for the $i$ th stage, $t \in\left[t_{0}^{i}, t_{1}^{i}\right], i=1, \ldots, N$, the optimal control law, the corresponding global minimizer, the optimal trajectory, and the minimum performance index satisfy the same corresponding equations in Theorem 2 , except that $t_{1}^{N}=\infty$ and $Q^{i}=0$ for all $i=1, \ldots, N$.

Proof: This theorem obviously holds with Theorem 2. Notice that, for the $N$ th stage, the controllable criterion can ensure the existence of the limit value of $\pi^{N}\left(t, t_{1}\right)$; i.e., $\bar{\pi}^{N}(t)=\lim _{t_{1} \rightarrow \infty} \pi^{N}\left(t, t_{1}\right)$ exists for all $t$, and $\bar{\pi}^{N}(t)$ is still the symmetric positive semidefinite solution of the segmental Riccati-like DE in (16) [20].

For the time-invariant case, the pair $\left(H\left(X_{0}\right) A, H\left(X_{o}\right) B\right) \mathrm{CC}$ is equivalent to $\operatorname{rank}\left[\lambda I_{n}-H\left(X_{o}\right) A, H\left(X_{o}\right) B\right]=n, \forall \mathcal{C}$ and this condition, by Lemma 3 , can be satisfied if $\left(A_{i}, B_{i}\right)$ is CC, for all $i=1, \ldots, r$. So, we need the following assumption as the prerequisite for the optimal controller design in the timeinvariant infinite-horizon case.

Assumption 2: $\left(A_{i}, B_{i}\right)$ is $\mathrm{CC}$, for all $i=1, \ldots, r$.

Theorem 7 (Time-Invariant): Consider the time-invariant fuzzy system and fuzzy controller described, respectively, by (1) and (2) with $L=C^{t} C$ in (8). If $N>\bar{N},\left(A_{i}, B_{i}\right)$ is CC and $\left(A_{i}, C\right)$ is $\mathrm{CO}$, for all $i=1, \ldots, r$ then

2)

$$
\begin{aligned}
&\left(X_{\infty}^{*}(t), R_{\infty}^{*}(t)\right)=\left(X_{\infty}^{i^{*}}(t), R_{\infty}^{i^{*}}(t)\right) \\
& \forall t \in\left[t_{0}^{i}, t_{1}^{i}\right], \quad t_{0}^{1}=t_{0}, \\
& t_{1}^{N}=\infty, \quad i=1, \ldots, N
\end{aligned}
$$

where $R_{\infty}^{i *}(t)$ is the $i$ th-stage asymptotically optimal control law in (24) and $X_{\infty}^{i^{*}}(t)$ is the corresponding asymptotically optimal trajectory in (26), where $\pi_{\infty}^{i}$ is the unique symmetric positive semidefinite solution of the SSRE in (23);

3) the minimum performance index is

$$
\min _{R_{\left[t_{0}, \infty\right)}} J(R(\cdot))=\sum_{i=1}^{N}\left[X^{i^{*^{t}}}\left(t_{0}^{i}\right) \pi_{\infty}^{i} X^{i^{*}}\left(t_{0}^{i}\right)\right] .
$$

Proof: This theorem obviously holds with Theorem 3.

\section{Stability AND GAin MARgin}

In this section, we shall show that the control law resulting from an infinite-horizon performance index cannot only asymp- totically and exponentially stabilize the fuzzy system, but also form a closed-loop fuzzy system with any desired degree of stability. We also concern with the range of the feedback gain gain margin to which we can increase under the stability consideration.

\section{A. Global Stability}

As remarked earlier, the whole optimal trajectory is decomposed into $N$ segments and, more, if each fuzzy subsystem in (1) is well-behaved (CC and $\mathrm{CO}$ ) and $N>\bar{N}$, then each segment can be described by its corresponding asymptotically optimal trajectory during the same period of this segment, i.e.,

$$
X_{\infty}^{*}(t) \equiv X^{i^{*}}(t) \equiv X_{\infty}^{i^{*}}(t), \quad \forall t \in\left[t_{0}^{i}, t_{1}^{i}\right]
$$

where $i=1, \ldots, N$ and $t_{0}^{1}=t_{0}, t_{1}^{N}=t_{1}, t_{0}^{i}=t_{1}^{i-1}, i=$ $2, \ldots, N$; that is, the behavior of the closed-loop fuzzy system can be captured by the corresponding asymptotically behavior of these $N$ segments.

Theorem 8: For the time-invariant fuzzy system and fuzzy controller described, respectively, in (1) and (2) with $L=C^{t} C$ in (8). If $N>\bar{N},\left(A_{i}, B_{i}\right)$ is CC, and $\left(A_{i}, C\right)$ is CO for $i=$ $1, \ldots, r$, then

2) the optimal closed-loop fuzzy system

$$
\dot{X}_{\infty}^{*}(t)=\left[H_{i} A-H_{i} B B^{t} H_{i}^{t} \pi_{\infty}^{i}\right] X_{\infty}^{*}(t), \quad t \in\left[t_{0}^{i}, t_{1}^{i}\right]
$$

where $i=1, \ldots, N, t_{0}^{1}=t_{0}, t_{1}^{N}=\infty, t_{0}^{i}=t_{1}^{i-1}$, $i=2, \ldots, N$ is exponentially stable;

3) the total energy of system output is finite, i.e., $\int_{t_{0}}^{\infty}\left\|Y^{*}(t)\right\|^{2} d t<\infty$.

Proof:

2) Recall that $X_{\infty}^{i^{*}}(\cdot)$ is the $i$ th-stage asymptotically optimal trajectory of the quadratic optimal control problem, i.e., minimizing the performance index $J_{\infty}^{i}(R(\cdot))$ in (25) with respect to the dynamical fuzzy system in (22). Moreover, $\left(A_{i}, B_{i}\right) \mathrm{CC}$ and $\left(A_{i}, C\right) \mathrm{CO}, \forall i=1, \ldots, r$, guarantees from the proof of Lemma $5\left(H_{i} A, H_{i} B\right) \mathrm{CC}$ and $\left(H_{i} A, C\right) \mathrm{CO}, \forall i=1, \ldots, N$. Hence, we know from Lemma 5 the $i$ th-stage asymptotically optimal trajectory

$$
\dot{X}_{\infty}^{i^{*}}(t)=\left[H_{i} A-H_{i} B B^{t} H_{i}^{t} \pi_{\infty}^{i}\right] X_{\infty}^{i^{*}}(t), \quad t \in\left[t_{0}^{i}, \infty\right)
$$

is asymptotically and exponentially stable, i.e., $\sigma\left[H_{i} A-H_{i} B B^{t} H_{i}^{t} \pi_{\infty}^{i}\right] \subset \mathcal{C}_{-}^{o}$, where $\mathcal{C}_{-}^{o}$ denotes the open left-half plane of complex space. Hence, via (39), the optimal trajectory described by (40) is asymptotically and exponentially stable since the eigenvalues of the system matrix characterizing the dynamical behavior of each segment are all located on the left-half plane of complex space.

$$
J^{i}(R(\cdot))= \begin{cases}\int_{t_{i}^{i}}^{t_{i}^{i}}\left(X^{t}(t) L(t) X(t)+R^{t}(t) W^{t}(Y(t)) W(Y(t)) R(t)\right) d t, \quad \forall t \in\left[t_{0}^{i}, t_{1}^{i}\right], \quad i=1, \ldots, N-1 \\ \int_{t_{0}^{N}}^{\infty}\left(X^{t}(t) L(t) X(t)+R^{t}(t) W^{t}(Y(t)) W(Y(t)) R(t)\right) d t, \quad \forall t \in\left[t_{0}^{N}, \infty\right), \quad i=N\end{cases}
$$


3) From (7)

$$
\begin{aligned}
\int_{t_{0}}^{\infty}\left\|Y^{*}(t)\right\|^{2} d t & \leq \int_{t_{0}}^{\infty}\|C\|^{2}\left\|X_{\infty}^{*}(t)\right\|^{2} d t \\
& =\sum_{i=1}^{N} \int_{t_{0}^{i}}^{t_{1}^{i}}\|C\|^{2}\left\|X_{\infty}^{i^{*}}(t)\right\|^{2} d t .
\end{aligned}
$$

From (1) of this proof, the $i$ th-stage asymptotically optimal trajectory $X_{\infty}^{i^{*}}(\cdot)$ is exponentially stable. The term exponentially stable means uniformly asymptotically stable in the stability concept [22], which means that for all $X\left(t_{0}^{i}\right) \in \Re^{n}$ and $t_{0}^{i} \in \Re_{+}, X_{\infty}^{i^{*}}(t)$ satisfies the following two properties.

2) The range of mapping from $t$ to $X_{\infty}^{i^{*}}(t)$ is bounded on $t>0$ uniformly, i.e.,

$$
\exists k<\infty \text { s.t. }\left\|X_{\infty}^{i^{*}}(t)\right\|<k, \quad \forall t>0 .
$$

3) The range of mapping from $t$ to $X_{\infty}^{i *}(t)$ tends to zero as $t \longrightarrow \infty$ uniformly, i.e.,

$\forall \epsilon>0, \quad \exists T(\epsilon)>0$ s.t. $\left\|X_{\infty}^{i^{*}}(t)\right\| \leq \epsilon, \quad \forall t>T(\epsilon)$.

Assume $T(\epsilon)$ is located in the $N_{o}$-th stage, i.e., $t_{0}^{N_{o}} \leq$ $T(\epsilon) \leq t_{1}^{N_{o}}$. Then, (43) becomes

$$
\begin{aligned}
\int_{t_{0}}^{\infty} & \left\|Y^{*}(t)\right\|^{2} d t \\
\leq & \sum_{i=1}^{N_{o}-1} \int_{t_{0}^{i}}^{t_{1}^{i}}\|C\|^{2}\left\|X_{\infty}^{i^{*}}(t)\right\|^{2} d t \\
& +\int_{t_{0}^{N_{o}}}^{T(\epsilon)}\|C\|^{2}\left\|X_{\infty}^{i^{*}}(t)\right\|^{2} d t \\
& +\int_{T(\epsilon)}^{t_{1}^{N_{o}}}\|C\|^{2}\left\|X_{\infty}^{i^{*}}(t)\right\|^{2} d t \\
& +\sum_{N_{o}+1}^{N} \int_{t_{0}^{i}}^{t_{1}^{i}}\|C\|^{2}\left\|X_{\infty}^{i^{*}}(t)\right\|^{2} d t
\end{aligned}
$$

where $t_{1}^{N}=\infty$. We know that the first two terms are finite and the others are infinitesimal and, thereupon, the total energy of system output is absolutely finite.

The stability of the closed-loop fuzzy system in time-varying case can still be ensured if the corresponding asymptotically optimal trajectory of each segment is exponentially stable.

\section{B. Stabilization to Any Desired Degree}

This section shows that the resultant closed-loop fuzzy system has a degree of stability of at least some prescribed constant $\alpha, \alpha>0$, which means that the state approaches zero at least by the rate of $e^{-\alpha t}$. Of course, the larger the desired degree of stability is, the more stable the closed-loop fuzzy system is. However, a high degree of stability may only be achieved at the expense of excessive control energy consumption. Two lemmas are derived here to deduce this interesting property.

Lemma 6: For a system $R=[A, B, C]: \dot{X}(t)=A X(t)+$ $B u(t), Y(t)=C X(t)$, where $A, B$ and $C$ are $n \times n, n \times m$, and $n^{\prime} \times n$ matrices, $(A, B) \mathrm{CC}$ is equivalent to $\left(\alpha I_{n}+A, B\right)$ $\mathrm{CC}$ for any complex value $\alpha$.
Proof: 1) $(A, B)$ is $\mathrm{CC}$ if and only if $\operatorname{rank}\left[B \quad A B \cdots A^{n-1} B\right]=n$. Let $\hat{B} \triangleq e^{-\alpha t} B$. Then we have $\operatorname{rank}\left[\begin{array}{lll}\hat{B} & A \hat{B} \cdots A^{n-1} \hat{B}\end{array}\right]=\operatorname{rank}\left[\begin{array}{ll}B & \left.A B \cdots A^{n-1} B\right]\end{array}\right]$ which means $(A, B)$ is $\mathrm{CC}$ if and only if $(A, \hat{B})$ is $\mathrm{CC}$; 2) Now, consider two systems: $\dot{X}=A X+\hat{B} u$ and $\dot{\hat{X}}=\left(\alpha I_{n}+A\right) \hat{X}+B u$. Obviously, they are related by a nonsingular linear transformation $e^{\alpha t}$. Therefore, $(A, \hat{B})$ is CC if and only if $\left(\alpha I_{n}+A, B\right)$ is CC for any complex value $\alpha$. From 1) and 2), we conclude that Lemma 6 holds.

Lemma 7: For a system $R=[A, B, C],(A, C) \mathrm{CO}$ is equivalent to $\left(\alpha I_{n}+A, C\right) \mathrm{CO}$ for any complex value $\alpha$.

Proof: The proof is similar to the proof of Lemma 6.

Theorem 9: Consider the time-invariant fuzzy system and fuzzy controller described, respectively, by (1) and (2) with $L=$ $C^{t} C$ in (8). If $N>\bar{N},\left(A_{i}, B\right)$ is $\mathrm{CC}$ and $\left(A_{i}, C\right)$ is $\mathrm{CO}$ for all $i=1, \ldots, r$, then the fuzzy system can be stabilized to any desired degree of stability, i.e., the optimal trajectory $X_{\infty}^{*}(\cdot)$ will approach zero at least by the rate of $e^{-\alpha t}$, where $\alpha$ is any positive real value.

Proof:

2) As we know, for $N>\bar{N}$ and well-behaved fuzzy subsystems, the behavior of $X_{\infty}^{*}(\cdot)$ is fully described by $X_{\infty}^{i^{*}}(\cdot)$, $i=1, \ldots, N$. Hence, we now pay attention to such quadratic optimal control problem: minimizing the performance index in (25) with respect to the linear time-invariant fuzzy system in (22). Let $A i, B i$ and $u$ denote, respectively, $H_{i} A, H_{i} B$ and $W_{i} R$. Then we have the following optimization problem:

$$
\begin{gathered}
\min \int_{t_{0}^{i}}^{\infty}\left[X^{t}(t) L X(t)+u^{t}(t) u(t)\right] d t \\
\text { w.r.t. }\left\{\begin{array}{l}
\dot{X}(t)=A i X(t)+B i u(t), \\
Y(t)=C X(t) .
\end{array}\right.
\end{gathered}
$$

Let $\hat{X}(t)=e^{\alpha t} X(t), \hat{Y}(t)=e^{\alpha t} Y(t)$ and $\hat{u}(t)=$ $e^{\alpha t} u(t)$. Then (43) can be rewritten as

$$
\begin{gathered}
\min \int_{t_{0}^{i}}^{\infty} e^{-2 \alpha t}\left[\hat{X}^{t}(t) L \hat{X}(t)+\hat{u}^{t}(t) \hat{u}(t)\right] d t \\
\text { w.r.t. }\left\{\begin{array}{l}
\dot{\hat{X}}(t)=\left(\alpha I_{n}+A i\right) \hat{X}(t)+B i \hat{u}(t), \\
\hat{Y}(t)=C \hat{X}(t) .
\end{array}\right.
\end{gathered}
$$

3) From Lemmas 6 and 7 , we know that $(A i, B)$ is $\mathrm{CC}$ and $(A i, C)$ is $\mathrm{CO}, \forall i=1, \ldots, r$, if and only if $\left(\alpha I_{n}+\right.$ $A i, B i)$ is $\mathrm{CC}$ and $\left(\alpha I_{n}+A i, C\right)$ is $\mathrm{CO}, \forall i=1, \ldots, N$. Hence, based on the theorems in [19], [23], [24], the optimal fuzzy control law for the modified fuzzy system in the above is $\hat{u}_{\infty}^{i^{*}}(t)=-B i^{t} \hat{\pi}_{\infty}^{i}(\alpha) \hat{X}_{\infty}^{i *}(t), i=1, \ldots, N$, where $\hat{\pi}_{\infty}^{i}(\alpha)$ is the positive semidefinite solution of the modified SSRE

$$
\begin{gathered}
\left(\alpha I_{n}+H_{i} A\right)^{t} K(\alpha)+K(\alpha)\left(\alpha I_{n}+H_{i} A\right) \\
-K(\alpha) H_{i} B B^{t} H_{i}^{t} K(\alpha)+L=0
\end{gathered}
$$

and the modified fuzzy system is asymptotically stable, i.e., $\hat{X}_{\infty}^{i *}(t) \rightarrow 0, \forall i=1, \ldots, N$ as $t \rightarrow \infty$. Then, $X_{\infty}^{i^{*}}(t), \forall i=1, \ldots, N$, decays faster than $e^{-\alpha t}$ as $t \rightarrow$ $\infty$ since $\hat{X}_{\infty}^{i^{*}}(t)=e^{\alpha t} X_{\infty}^{i^{*}}(t)$. Hence, by (39), $X_{\infty}^{*}(\cdot)$ will approach zero at least by the rate of $e^{-\alpha t}$. 


\section{Gain Margin}

In the remainder of Section IV, we examine another characteristic gain margin of the resultant closed-loop fuzzy system. For the time-invariant well-behaved fuzzy subsystems, if $N>\bar{N}$, then $X_{\infty}^{*}(t)$ is coincident with $X_{\infty}^{i^{*}}(t)$, for all $t \in\left[t_{0}^{i}, t_{1}^{i}\right]$, where $i=1, \ldots, N$. Therefore, we can only discuss the asymptotically behavior of the dynamical fuzzy system of each stage and then turns it into the behavior of the entire dynamical fuzzy system via (39). From Lemma 5, the designed ith-stage asymptotically global minimizer is

$$
u_{\infty}^{i^{*}}(t)=-B^{t} H_{i}^{t} \pi_{\infty}^{i} X_{\infty}^{i^{*}}(t), \quad t \in\left[t_{0}^{i}, \infty\right) .
$$

In order to measure the gain margin, we consider the following corresponding controller:

$$
u_{\infty}^{i}(t)=-\beta B^{t} H_{i}^{t} \pi_{\infty}^{i} X_{\infty}^{i}(t), \quad \beta \geq 1, \quad t \in\left[t_{0}^{i}, \infty\right) .
$$

The gain margin of the ith-stage closed-loop fuzzy system is defined as the amount by which $\beta$ can be increased until the system becomes unstable [20]. Now, let

$$
v_{\infty}^{i}(t) \triangleq u_{\infty}^{i}(t) / \beta=-B^{t} H_{i}^{t} \pi_{\infty}^{i} X_{\infty}^{i}(t)
$$

and then we have

$$
\begin{aligned}
J\left(u_{\infty}^{i}(\cdot)\right) & =\int_{t_{0}^{i}}^{\infty}\left(X_{\infty}^{i^{t}} L X_{\infty}^{i}+u_{\infty}^{i^{t}} u_{\infty}^{i}\right) d t \\
& =\int_{t_{0}^{i}}^{\infty}\left(X_{\infty}^{i^{t}} L X_{\infty}^{i}+\beta^{2} v_{\infty}^{i^{t}} v_{\infty}^{i}\right) d t .
\end{aligned}
$$

We further consider

$$
J\left(v_{\infty}^{i}(\cdot)\right)=\int_{t_{0}^{i}}^{\infty}\left(q X_{\infty}^{i^{t}} L X_{\infty}^{i}+v_{\infty}^{i^{t}} v_{\infty}^{i}\right) d t, \quad q>0 .
$$

Notice that $J\left(u_{\infty}^{i}(\cdot)\right)=\beta^{2} J\left(v_{\infty}^{i}(\cdot)\right)$ and $q=1 / \beta^{2}$. Comparing (50) to (49), we find that the larger the $\beta$ is, the smaller the $q$ is, which means that when $q$ goes to zero, the gain margin of the $i$ th-stage closed-loop fuzzy system becomes infinite.

It is realizable to include $q$ into the state penalty matrix $L$. From Lemma 5, for any $q>0$, the global minimizer becomes

$$
v_{\infty}^{i^{*}}(t)=-B^{t} H_{i}^{t} \hat{\pi}_{\infty}^{i}(q) X_{\infty}^{i^{*}}(t)
$$

where $\hat{\pi}_{\infty}^{i}(q)$ satisfies the modified SSRE

$$
A^{t} H_{i}^{t} K(q)+K(q) H_{i} A+q L-K(q) H_{i} B B^{t} H_{i}^{t} K(q)=0
$$

where $K(q)$ is the dependent variable of the algebraic equation. We now first cite some important results in control theory [25], [26] and apply them to the $i$ th-stage closed-loop fuzzy system. We shall then find the gain margin of the entire closed-loop fuzzy system.

Proposition 1 [25], [26]: Consider the infinite-horizon optimal control problem as follows: Given a linear time-invariant system $\dot{X}(t)=A X(t)+B u(t)$, find an optimal controller $u^{*}(t)$ to minimize $J(u(\cdot))=\int_{0}^{\infty}\left[X^{t}(t) L X(t)+u^{t}(t) u(t)\right] d t$ with $Y(t)$ denoting the system output, where $Y(t)=C X(t)$ and $L=C^{t} C$. Now, if $(A, B)$ is $\mathrm{CC}$ and $(A, C)$ is $\mathrm{CO}$, then

2) there exists an unique symmetric positive semidefinite solution denoted by $\hat{\pi}_{\infty}(q)$ of the modified SSRE

$$
A^{t} K(q)+K(q) A+q L-K(q) B B^{t} K(q)=0
$$

3) when $q$ goes to zero, which is the case that the closed-loop system possesses an infinite gain margin, the limit value of $\hat{\pi}_{\infty}(q)$ exists and is equal to $\hat{\pi}_{\infty}(0)$;

4) we can find an optimal control law, $u^{*}(t)=$ $-B^{t} \pi_{\infty} X^{*}(t)$, where $\pi_{\infty}$ is the positive semidefinite solution of the SSRE $A^{t} K+K A-K B B^{t} K+L=0$ and the resultant closed-loop system possesses an infinite gain margin, i.e., the modified closed-loop system $\left[A-\beta B B^{t} \hat{\pi}_{\infty}(q)\right]$ is always stable for any $\beta \geq 1$, where $q=1 / \beta^{2}$ and $\hat{\pi}_{\infty}(q)$ is the positive semidefinite solution of the modified SSRE in (52);

$5)$ moreover, for any fixed $\beta$, the enlarged controller, $\hat{u}^{*}(t)=-\beta B^{t} \hat{\pi}_{\infty}(q) X^{*}(t)$ can still stabilize the modified closed-loop system to any desired degree of stability, i.e., all the eigenvalues of $A-\beta B B^{t} \hat{\pi}_{\infty}(\alpha, q)$ have real parts smaller than $-\alpha$, where $\alpha$ could be any positive real number and $\hat{\pi}_{\infty}(\alpha, q)$ is the positive semidefinite solution of the modified SSRE

$$
\begin{gathered}
\left(\alpha I_{n}+A\right)^{t} K(\alpha, q)+K(\alpha, q)\left(\alpha I_{n}+A\right) \\
\quad+q L-K(\alpha, q) B B^{t} K(\alpha, q)=0
\end{gathered}
$$

where $K(\alpha, q)$ is the dependent variable of the algebraic equation.

Grounding on this proposition, the following fascinating fact can be elicited.

Theorem 10: Consider the time-invariant fuzzy system and fuzzy controller described, respectively, by (1) and (2) with $L=$ $C^{t} C$ in (8). If $N>\bar{N},\left(A_{i}, B_{i}\right)$ is $\mathrm{CC}$ and $\left(A_{i}, C\right)$ is $\mathrm{CO}$ for all $i=1, \ldots, r$, then

2) we can find a global minimizer

$$
u_{\infty}^{*}(t)=-B^{t} H_{i}^{t} \pi_{\infty}^{i} X_{\infty}^{*}(t), \quad t \in\left[t_{0}^{i}, t_{1}^{i}\right]
$$

where $i=1, \ldots, N ; t_{0}^{1}=t_{0}, t_{1}^{N}=\infty, t_{0}^{i}=t_{1}^{i-1}$, $i=2, \ldots, N$; and $\pi_{\infty}^{i}$ is the positive semidefinite solution of the SSRE in (23) and the resultant closed-loop fuzzy system possesses an infinite gain margin, i.e., the modified closed-loop fuzzy system $\left[H_{i} A-\beta H_{i} B B^{t} H_{i}^{t} \hat{\pi}_{\infty}^{i}(q)\right], t \in\left[t_{0}^{i}, t_{1}^{i}\right], i=1, \ldots, N$, is always stable for any $\beta \geq 1$, where $q=1 / \beta^{2}$ and $\hat{\pi}_{\infty}^{i}(q)$ is the positive semidefinite solution of the modified SSRE

$$
A^{t} H_{i}^{t} K(q)+K(q) H_{i} A+q L-K(q) H_{i} B B^{t} H_{i}^{t} K(q)=0 .
$$

3) moreover, for any fixed $\beta$ the enlarged controller $\hat{u}_{\infty}^{*}(t)=-\beta B^{t} H_{i}^{t} \hat{\pi}_{\infty}^{i}(q) X_{\infty}^{*}(t), t \in\left[t_{0}^{i}, t_{1}^{i}\right]$, $i=1, \ldots, N$ can still stabilize the modified closed-loop system to any desired degree of stability, i.e., all the eigenvalues of $\left[H_{i} A-\beta H_{i} B B^{t} H_{i}^{t} \hat{\pi}_{\infty}^{i}(\alpha, q)\right], t \in\left[t_{0}^{i}, t_{1}^{i}\right]$, $i=1, \ldots, N$, have real parts smaller than $-\alpha$, where $\alpha$ could be any positive real number and $\hat{\pi}_{\infty}^{i}(\alpha, q)$ is the positive semidefinite solution of the modified SSRE

$$
\begin{gathered}
\left(\alpha I_{n}+H_{i} A\right)^{t} K(\alpha, q)+K(\alpha, q)\left(\alpha I_{n}+H_{i} A\right) \\
\quad+q L-K(\alpha, q) H_{i} B B H_{i}^{t} K(\alpha, q)=0
\end{gathered}
$$

where $K(\alpha, q)$ is the dependent variable of the algebraic equation. 
Proof: From the proof of Lemma 5, $\left(A_{i}, B_{i}\right) \mathrm{CC}$, and $\left(A_{i}, C\right) \mathrm{CO}, \forall i=1, \ldots, r$, ensures $\left(H_{i} A, H_{i} B\right) \mathrm{CC}$, and $\left(H_{i} A, C\right) \mathrm{CO}, \forall i=1, \ldots, N$, which meets the condition in Proposition 1. Hence, we shall, via Proposition 1, obtain the asymptotically optimal solution for each segment and then, via (39) $\left(X_{\infty}^{*}(t) \equiv X^{i^{*}}(t) \equiv X_{\infty}^{i^{*}}(t), \forall t \in\left[t_{0}^{i}, t_{1}^{i}\right]\right)$, get the whole optimal solution.

2) Let $A i, B i$ and $u$ denote $H_{i} A, H_{i} B$ and $W_{i} R$, respectively. We know, from 3) in Proposition 1 , that the asymptotically global minimizer is $u_{\infty}^{i^{*}}(t)=-B^{t} H_{i}^{t} \pi_{\infty}^{i} X_{\infty}^{i^{*}}(t), t \in\left[t_{0}^{i}, \infty\right)$ and the modified closed-loop fuzzy system of the $i$ th stage

$$
\begin{array}{r}
\dot{X}_{\infty}^{i^{*}}(t)=\left(H_{i} A-\beta H_{i} B B^{t} H_{i}^{t} \hat{\pi}_{\infty}^{i}(q)\right) X_{\infty}^{i^{*}}(t) \\
t \in\left[t_{0}^{i}, \infty\right), \quad \forall i=1, \ldots, N
\end{array}
$$

is always stable for any $\beta \geq 1$, i.e., $\sigma\left[H_{i} A-\right.$ $\left.\beta H_{i} B B^{t} H_{i}^{t} \hat{\pi}_{\infty}(q)\right] \subset \mathcal{C}_{-}^{o}$, where $q=1 / \beta^{2}$ and $\mathcal{C}_{-}^{o}$ denotes the open left-half plane of complex space. By (39), we know that the global minimizer is $u_{\infty}^{*}(t)$ in (53) and the modified closed-loop fuzzy system is

$$
\begin{array}{r}
\dot{X}_{\infty}^{*}(t)=\left(H_{i} A-\beta H_{i} B B^{t} H_{i}^{t} \hat{\pi}_{\infty}^{i}(q)\right) X_{\infty}^{*}(t) \\
t \in\left[t_{0}^{i}, t_{1}^{i}\right]
\end{array}
$$

where $i=1, \ldots, N$ and $t_{0}^{1}=t_{0}, t_{1}^{N}=\infty, t_{0}^{i}=t_{1}^{i-1}$, $i=2, \ldots, N$. Since no matter how large $\beta$ is, the spectrum of system matrix, which characterizes the dynamical behavior of each segment of the modified optimal trajectory, is always located in the left half plane of complex space, the resultant closed-loop fuzzy system posses infinite gain margin.

3) Then, for any given gain margin $\beta$, we know, from Proposition 1, 4), all the eigenvalues of $\left[H_{i} A-\beta H_{i} B B^{t} H_{i}^{t} \hat{\pi}_{\infty}^{i}(\alpha, q)\right], i=1, \ldots, N$ have real parts smaller than $-\alpha$, which means that the $i$ th-stage modified closed-loop fuzzy system

$$
\begin{array}{r}
\dot{X}_{\infty}^{i^{*}}(t)=\left(H_{i} A-\beta H_{i} B B^{t} H_{i}^{t} \hat{\pi}_{\infty}^{i}(\alpha, q)\right) X_{\infty}^{i^{*}}(t) \\
t \in\left[t_{0}^{i}, \infty\right), i=1, \ldots, N
\end{array}
$$

decays faster than $e^{-\alpha t}$ as $t$ approaches infinity. Since the modified closed-loop fuzzy system via (39) is

$$
\begin{array}{r}
\dot{X}_{\infty}^{*}(t)=\left(H_{i} A-\beta H_{i} B B^{t} H_{i}^{t} \hat{\pi}_{\infty}^{i}(\alpha, q)\right) X_{\infty}^{*}(t) \\
t \in\left[t_{0}^{i}, t_{1}^{i}\right]
\end{array}
$$

where $i=1, \ldots, N$ and $t_{0}^{1}=t_{0}, t_{1}^{N}=\infty, t_{0}^{i}=t_{1}^{i-1}$, $i=2, \ldots, N$, we conclude that the above closed-loop fuzzy system decays faster than $e^{-\alpha t}$ as $t$ approaches infinity, too.

\section{PRACTICAL APPLICATION}

In this section, we propose an algorithm to implement the theorems in Section III and consider a simple nonlinear massspring damper mechanical system to illustrate the proposed optimal fuzzy control scheme.

\section{A. Dynamic Decomposition Algorithm}

We shall propose a procedure to check the two inequalities in (17) and (18), which can ensure the almost-invariant-mem- bership-function criteria during a whole single stage. Now, by denoting the time-dependence as a lower index (i.e., $X_{\tau}^{*}$ for $X^{*}(\tau)$ ) and substituting $t_{0}^{i}$ by $t$ for notation simplification, we can rewrite (17) as follows:

$$
\begin{aligned}
\left\|\frac{d H\left(X^{*}(\tau)\right)}{d \tau}\right\|_{\tau=t}= & \|\left[\nabla_{X_{\tau}^{*}}^{t} h_{1}\left(X_{\tau}^{*}\right) \cdot \frac{d X_{\tau}^{*}}{d \tau}\right. \\
& \cdot I_{n}, \ldots, \nabla_{X_{\tau}^{*}}^{t} h_{r}\left(X_{\tau}^{*}\right) \cdot \frac{d X_{\tau}^{*}}{d \tau} \cdot I_{n} \|_{\tau=t}
\end{aligned}
$$

where $\nabla_{X_{\tau}^{*}}^{t} h_{i}\left(X_{\tau}^{*}\right)=\left[\left(d h_{i}\left(X_{\tau}^{*}\right) / d x_{1}\right), \ldots,\left(d h_{i}\left(X_{\tau}^{*}\right) / d x_{n}\right)\right]$, $i=1, \ldots, r$. Substituting $P_{t}=K_{t} X_{t}^{*}$ into the TPBVP in Theorem 1, we have

$$
\begin{aligned}
-\dot{K}_{l}= & A_{l}^{t} H^{t}\left(X_{l}^{*}\right) K_{l}+K_{l} H\left(X_{l}^{*}\right) A_{l} \\
& -K_{l} H\left(X_{l}^{*}\right) B_{l} B_{l}^{t} H^{t}\left(X_{l}^{*}\right) K_{l}+L_{l}, \quad K_{t_{1}}=Q
\end{aligned}
$$

where $l \in\left[t_{0}, t_{1}\right]$. Though the entire backward Riccati DE in the above is unavailable in practice, the differential formula is always available for any time instant. In other words, at time instant $t$, we have

$$
\begin{aligned}
-\dot{K}_{t}= & A_{t}^{t} H^{t}\left(X_{t}^{*}\right) K_{t}+K_{t} H\left(X_{t}^{*}\right) A_{t} \\
& -K_{t} H\left(X_{t}^{*}\right) B_{t} B_{t}^{t} H^{t}\left(X_{t}^{*}\right) K_{t}+L_{t} .
\end{aligned}
$$

And, according to Lemma 3 , the finite-horizon optimal solution for the free-end problem is the same as the optimal solution of the infinite-horizon issue. Therefore, the solution $\pi_{t}$ for (62) is also the solution $\bar{\pi}_{t}$ of the following asymptotic Riccati-like equation

$$
\begin{aligned}
& A_{t}^{t} H^{t}\left(X_{t}^{*}\right) \bar{K}_{t}+\bar{K}_{t} H\left(X_{t}^{*}\right) A_{t} \\
& \quad-\bar{K}_{t} H\left(X_{t}^{*}\right) B_{t} B_{t}^{t} H^{t}\left(X_{t}^{*}\right) \bar{K}_{t}+L_{t}=0 .
\end{aligned}
$$

Also, we have

$$
\left.\frac{X_{\tau}^{*}}{d \tau}\right|_{\tau=t}=\left[H\left(X_{t}^{*}\right) A_{t}-H\left(X_{t}^{*}\right) B_{t} B_{t}^{t} H^{t}\left(X_{t}^{*}\right) \bar{\pi}_{t}\right] X_{t}^{*} .
$$

Hence, via (60) and (64), we can check the inequality in (17) at any time instant and the existence of $\bar{N}$ is guaranteed if the inequality holds at the starting time instant of every stage.

We propose a dynamic decomposition algorithm below to check the two inequalities in (17) and (18) and to find the proper time-period of each stage $\Delta t^{i}$ and also the value of $\bar{N}$ to ensure that the membership functions are almost invariant during a whole stage.

\section{Algorithm DDA: Dynamic Decomposition Algorithm}

Input: the initial chosen membership functions; initial state $X\left(t_{0}\right)$; time-increment $\kappa_{T}$; maximum number of design trials $n_{t}$.

Output: optimal controller $u^{*}(\cdot)$; optimal trajectory $X^{*}(\cdot)$; value of $\Delta t^{i}$; value of $\bar{N}(\bar{N}$ being initialized as $\bar{N}=0)$.

Step 0: (set threshold parameters) Set the default values of $\kappa_{H 1}$ and $\kappa_{H 2}$.

Step 1: (initial check)

$\operatorname{IF}\left(\left\|\left(d H\left(X^{*}(\tau)\right) / d \tau\right)\right\|_{\tau=t_{t_{0}}} \leq \kappa_{H_{1}}\right)$, THEN \{ go to Step 2 $\}$

ELSE \{choose a more smooth membership function and go back to Step 1, or break after $n_{t}$ times of failing trials. \} END 
Step 2: ( $t^{i}$ denoting the time-instant in the $i$ th stage, i.e., $t^{i} \in$ $\left[t_{0}^{i}, t_{1}^{i}\right] ; t^{i^{+}}=t^{i}+\kappa_{T}$.)

(a). Find out the solution $\bar{\pi}_{t^{i}}$ of (63) with the membership function $H\left(X_{t_{0}^{*}}^{*}\right)$.

(b). Calculate $u_{t^{i}}^{*}$ and $X_{t^{i}}^{*}$ by

$$
\begin{aligned}
u_{t^{i}}^{*}= & -B_{t^{i}}^{t} H^{t}\left(X_{t_{0}^{i}}^{*}\right) \bar{\pi}_{t^{i}} X_{t^{i}}^{*} \\
\dot{X}_{t^{i}}^{*}= & {\left[H\left(X_{t_{0}^{i}}^{*}\right) A_{t^{i}}-H\left(X_{t_{0}^{i}}^{*}\right)\right.} \\
& \left.B_{t^{i}} B_{t^{i}}^{t} H^{t}\left(X_{t_{0}^{i}}^{*}\right) \bar{\pi}_{t^{i}}\right] X_{t^{i}}^{*} .
\end{aligned}
$$

(c). IF $\left(\left\|H\left(X_{t^{i+}}^{*}\right)-H\left(X_{t_{0}^{i}}^{*}\right)\right\| \leq \kappa_{H_{2}}\right)$ THEN $\left\{t^{i}=t^{i^{+}}\right.$; go to $(a)$

END

(d). IF $\left(t^{i^{+}}=t_{1}\right)$ THEN $\left\{\triangle t^{N}=t_{1}-t_{0}^{i}\right.$; stop $\}$

END

Step 3: (find the starting point of the next stage $t_{0}^{i+1}=t_{1}^{i}$ ) $\operatorname{IF}\left(\left\|\left(d H\left(X^{*}(\tau)\right) / d \tau\right)\right\|_{\tau=t^{i}} \leq \kappa_{H_{1}}\right)$ THEN $\left\{t_{1}^{i}=t^{i} ; \triangle t^{i}=\right.$ $t_{1}^{i}-t_{0}^{i} ; \bar{N}=\bar{N}+1$; jump to Step 2

ELSE $\left\{\right.$ decrease $\kappa_{T}$ to get finer time-increment or choose another membership function and jump to Step 1 or break after $n_{t}$ times of failing trials.

END.

For the time-invariant finite-horizon (except the $N$ th stage) or infinite-horizon problem, the estimated optimal solutions $u_{t^{*}}^{*}$ in (65) and $X_{t^{i}}^{*}$ in (66) are also the optimal solutions $u_{\infty}^{i^{*}}(t)$ in (46) and $X_{\infty}^{i^{*}}(t)$ in (26), where the estimated $\bar{\pi}_{t^{i}}$ equals to $\pi_{\infty}^{i}$ in (23). As for the other case, we can obtain the optimal solutions $X^{i^{*}}(t)$ in (21) and $u^{i^{*}}(t)$ via (20) with the aid of the estimated $\triangle t^{i}$ and $\pi^{i}\left(t, t_{1}^{i}\right)$ in (16).

\section{B. Numerical Simulations}

In this section, we consider the optimal control of a massspring damper mechanical system to illustrate the proposed optimal fuzzy control scheme and its theoretic aspect. A simple nonlinear mass-spring damper mechanical system can be formulated as

$$
M \ddot{x}+g(x, \dot{x})+f(x)=\phi(\dot{x}) u
$$

where $M$ is the mass and $u$ is the force; $f(x)$ and $g(x, \dot{x})$ are the nonlinear or uncertain terms with respect to the spring and the damper, respectively, and $\phi(\dot{x})$ is the nonlinear term with respect to the input term. We make the same assumptions as Tanaka et al. [3] did and reformulate the system as

$$
\ddot{x}=-0.1 \dot{x}^{3}-0.02 x-0.67 x^{3}+u
$$

where $x \in\left[\begin{array}{ll}-1.5 & 1.5\end{array}\right]$ and $\dot{x} \in\left[\begin{array}{ll}-1.5 & 1.5\end{array}\right]$. Let $X(t)=[\dot{x}(t) x(t)]^{t}=\left[x_{1} x_{2}\right]^{t}$. The system in the above can be described by the following T-S type fuzzy model [3]:

$$
\begin{aligned}
& R^{i}: \text { If } x_{1}(t) \text { is } F_{1}^{i} \text { and } x_{2}(t) \text { is } F_{2}^{i}, \text { then } \\
& \dot{X}(t)=A_{i} X(t)+B_{i} u(t), \\
& Y(t)=C X(t), \quad i=1, \ldots, 4
\end{aligned}
$$

where the initial conditions are $X(0)=X_{0}$ and $Y(0)=C X_{0}$ with $C=I_{2}$ for every rule and the membership functions are chosen as $\mu_{F_{1}^{1}}=\mu_{F_{1}^{2}}=1-\left(x_{1}^{2}(t) / 2.25\right), \mu_{F_{1}^{3}}=\mu_{F_{1}^{4}}=$ $\left(x_{1}^{2}(t) / 2.25\right), \mu_{F_{2}^{1}}=\mu_{F_{2}^{3}}=1-\left(x_{2}^{2}(t) / 2.25\right), \mu_{F_{2}^{2}}=\mu_{F_{2}^{4}}=$ $\left(x_{2}^{2}(t) / 2.25\right), A_{1}=\left[\begin{array}{cc}0 & -0.02 \\ 1 & 0\end{array}\right], A_{2}=\left[\begin{array}{cc}-0.225 & -0.02 \\ 1 & 0\end{array}\right]$, $A_{3}=\left[\begin{array}{cc}0 & -1.5275 \\ 1 & 0\end{array}\right], A_{4}=\left[\begin{array}{cc}-0.225 & -1.5275 \\ 1 & 0\end{array}\right], B_{i}=$ $\left[\begin{array}{ll}1 & 0\end{array}\right]^{t}, i=1, \ldots, 4$. We further assume our fuzzy controller as$$
R^{i} \text { : If } x_{1}(t) \text { is } F_{1}^{i} \text { and } x_{2}(t) \text { is } F_{2}^{i} \text {, then } u(t)=r_{i}(t) \text {, }
$$$$
i=1, \ldots, 4 \text {. (70) }
$$

Accordingly, the firing-strength of the $i$ th rule is $\alpha_{i}(X(t))=$ $\mu_{F_{1}^{i}} \cdot \mu_{F_{2}^{i}}$ and the normalized firing-strength of the $i$ th rule is $h_{i}(X(t))=\alpha_{i}(X(t)) / \sum_{i=1}^{4} \alpha_{i}(X(t))$ for $i=1, \ldots, 4$. Therefore, the linear-like dynamical fuzzy system representation for the nonlinear mass-spring damper mechanical system is (7) with $A=\left[\begin{array}{cc}A_{1} & B_{1} \\ A_{2} \\ A_{3}\end{array}\right], B=\left[\begin{array}{l}B_{2} \\ B_{3}\end{array}\right], R=\left[\begin{array}{l}r_{2} \\ r_{3}\end{array}\right]$,

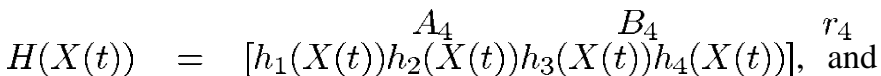
$W(Y(t))=\left[w_{1}(Y(t)) w_{2}(Y(t)) w_{3}(Y(t)) w_{4}(Y(t))\right]$.

For the finite-horizon free-end optimal control problem, the performance index is

$$
\begin{aligned}
J(R(\cdot))= & \int_{0}^{10}\left[X^{t}(t) L X(t)\right. \\
& \left.+R^{t}(t) W(Y(t))^{t} W(Y(t)) R(t)\right] d t \\
& +X^{t}(10) Q X(10)
\end{aligned}
$$

where $L=I_{2}$ and $Q=I_{2}$. As for the infinite-horizon case, the performance index is

$$
\begin{aligned}
J_{\infty}(R(\cdot))= & \int_{0}^{\infty}\left[X^{t}(t) L X(t)\right. \\
& \left.+R^{t}(t) W(Y(t))^{t} W(Y(t)) R(t)\right] d t
\end{aligned}
$$

Since the fuzzy subsystem is time-invariant and well-behaved, i.e., the fuzzy subsystem is $\mathrm{CC}$ and $\mathrm{CO}\left(\operatorname{rank}\left[A_{i} A_{i} B_{i}\right]=\right.$ 2 and $\operatorname{rank}\left[C^{t} A_{i}^{t} C^{t}\right]^{t}=2$ for $\left.i=1, \ldots, 4\right)$, the asymptotic Riccati-like equation in (63) becomes

$$
\begin{aligned}
& A^{t} H^{t}\left(X_{t}^{*}\right) \bar{K}_{t}+\bar{K}_{t} H\left(X_{t}\right) A \\
& \quad-\bar{K}_{t} H\left(X_{t}^{*}\right) B B^{t} H\left(X_{t}^{*}\right) \bar{K}_{t}+L=0 .
\end{aligned}
$$

Therefore, the steps $(a)$ and $(b)$ in Step 2 in algorithm DDA can be simplified as the following.

1) Find out the constant solution $\bar{\pi}$ of (73) with the membership function $H\left(X_{t_{0}^{*}}^{*}\right)$.

2) Calculate $u_{t^{i}}^{*}$ and $X_{t^{i}}^{*}$ by

$$
\begin{aligned}
& u_{t^{i}}^{*}=-B^{t} H^{t}\left(X_{t_{0}^{i}}^{*}\right) \bar{\pi} X_{t^{i}}^{*} \\
& \dot{X}_{t^{i}}^{*}=\left[H\left(X_{t_{0}^{i}}^{*}\right) A-H\left(X_{t_{0}^{i}}^{*}\right) B B^{t} H^{t}\left(X_{t_{0}^{i}}^{*}\right) \bar{\pi}\right] X_{t^{i}}^{*}
\end{aligned}
$$

Since the chosen membership functions are smooth Gaussian functions (see Fig. 1), we can efficiently obtain the optimal fuzzy controller with the aid of the DDA algorithm for determining appropriate segmentation under the almost-invariant membership-function criteria. For the initial state $X_{0}=[-1,1]^{t}$, the individual normalized firing strengths for the optimal trajectory (i.e., $h_{1}\left(X^{*}(t)\right), h_{2}\left(X^{*}(t)\right)$ and 
(a)

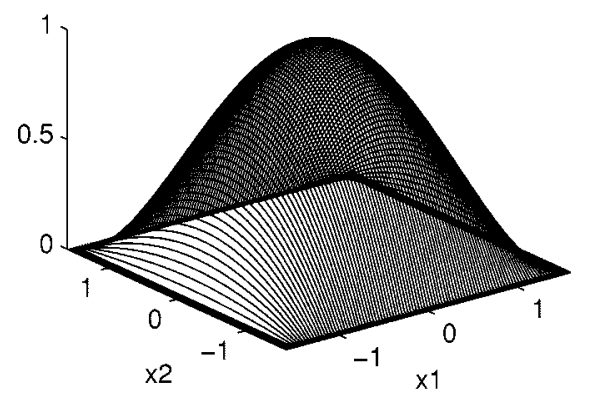

(c)

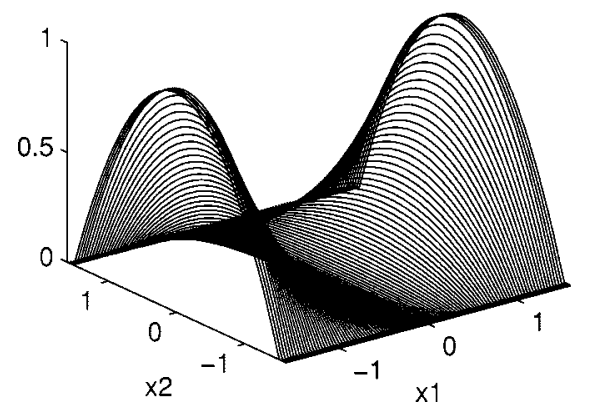

(b)

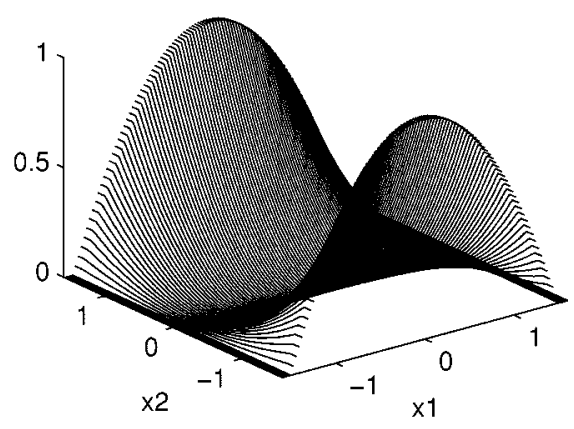

(d)

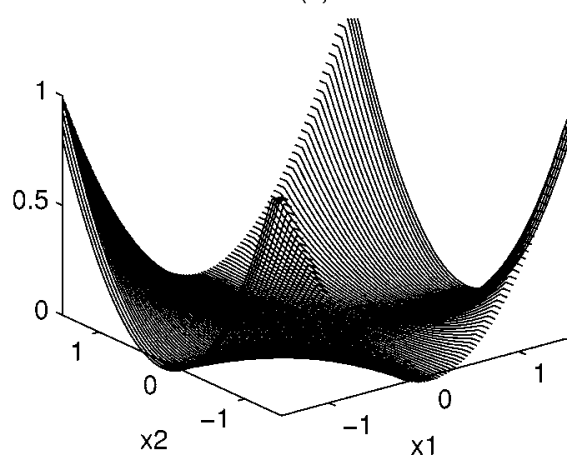

Fig. 1. Profile of the chosen membership functions for (a) $\alpha_{1}(X(t))$, (b) $\alpha_{2}(X(t))$, (c) $\alpha_{3}(X(t))$, and (d) $\alpha_{4}(X(t))$.

(a)

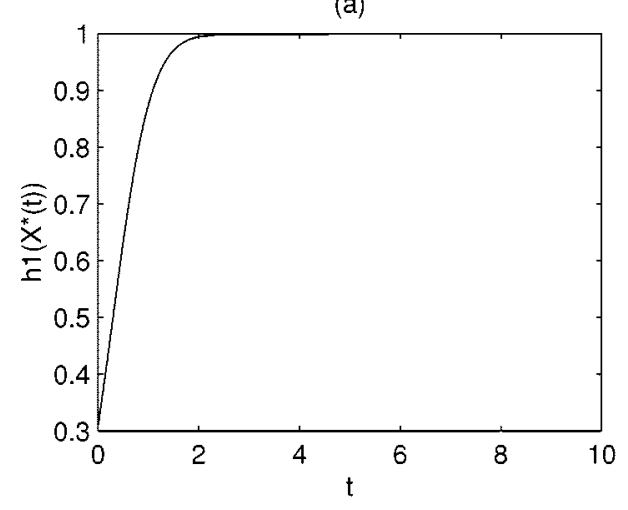

(c)

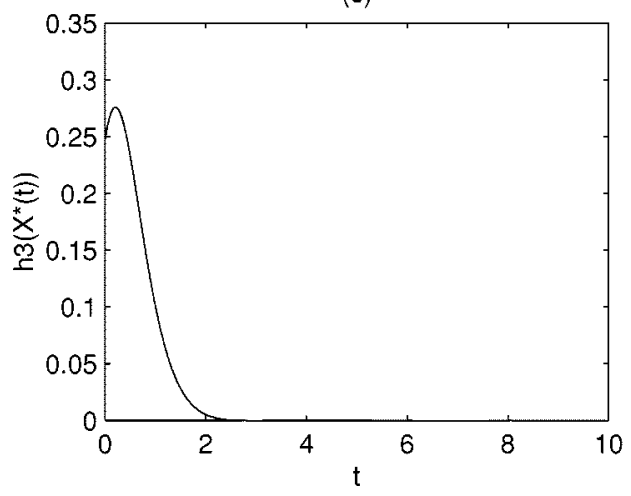

(b)

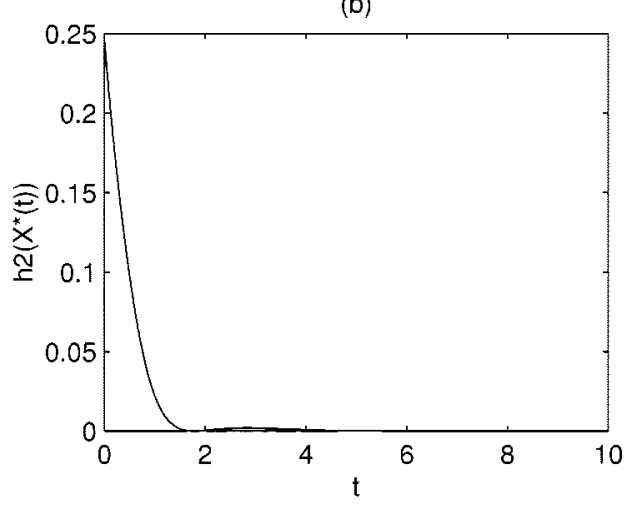

(d)

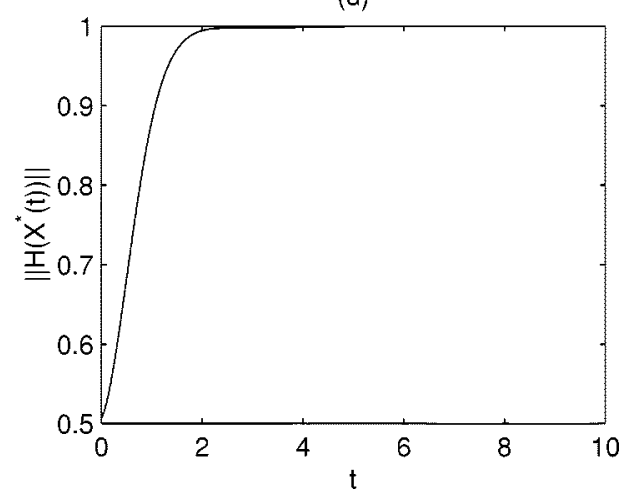

Fig. 2. Respectively, (a), (b), and (c) show the normalized firing strengths $h_{1}\left(X^{*}(t)\right), h_{2}\left(X^{*}(t)\right)$, and $h_{3}\left(X^{*}(t)\right)$, corresponding to the optimal trajectory. (d) The value of the norm of $H\left(X^{*}(k)\right)\left(X_{0}=[-1,1]^{t}\right)$. 

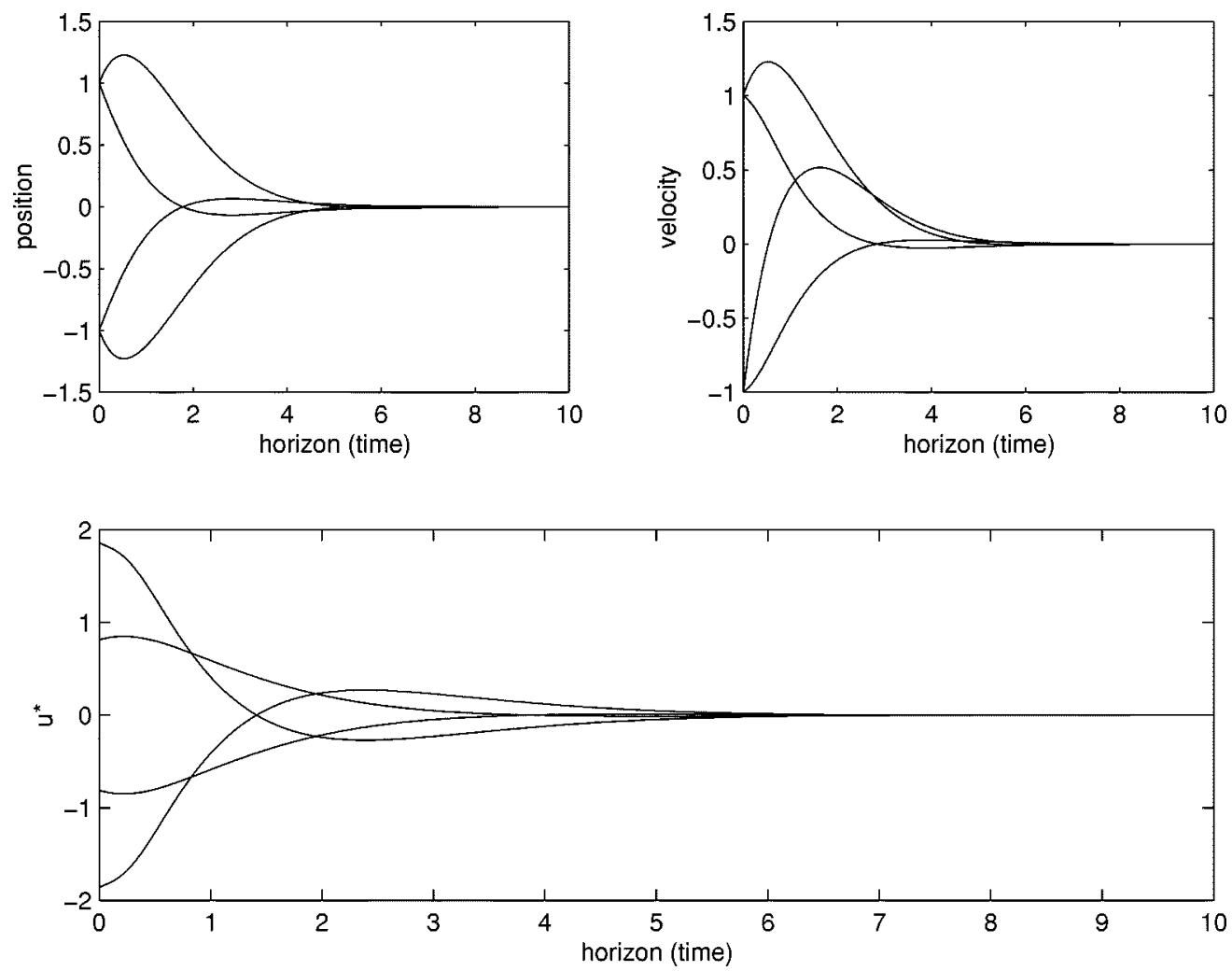

Fig. 3. Outputs of the optimal fuzzy controller $\left(u^{*}\right)$ and the state responses (position and velocity) of the continuous fuzzy system with the designed optimal fuzzy controller in the finite or infinite-horizon quadratic optimal control problem of Section V-B at the four initial conditions: $X(0)=(-1,-1)^{t} ;(-1,1)^{t}$; $(1,-1)^{t}$; and $(1,1)^{t}$.

$\left.h_{3}\left(X^{*}(t)\right)\right)$ and also the value of the norm of their synthetical matrix (i.e., $\left.\left\|H\left(X^{*}(t)\right)\right\|\right)$ are shown in Fig. 2. The outputs of the designed optimal fuzzy controller and the state responses of the resultant closed-loop fuzzy system in the finite-horizon case are shown in Fig. 3, which reveals that the designed optimal fuzzy controller can promptly push the simulated trunk-trailer system from various initial states to and stay at the desired state. Hence, the finite-state trajectory penalty vanishes and Theorems 3 and 5 are coincident.

\section{CONCLUSION}

The entire fuzzy system representation was proposed to maturate the formulation of the quadratic optimal fuzzy control problem and, further, a tricky unification of the individual matrices into synthetical matrices was proposed to generate a linear-like global system representation of continuous fuzzy systems. Based on this representation, the design scheme of global continuous optimal fuzzy controllers was derived theoretically. Furthermore, a multistage decomposition of optimization scheme was proposed to design the global optimal fuzzy controller more efficiently and keep the global optimality at the same time. Grounding on this efficient design scheme, several fascinating characteristics have been shown to exist in the resultant closed-loop continuous fuzzy system.

Overall, the fuzzy-blended entire fuzzy system is considered to formulate the quadratic optimal fuzzy control problem and the global optimal effect can then be achieved even though the chosen system model is composed of distributed rule-based fuzzy subsystems. This formation sheds light on the deadlock of the research of quadratic optimal fuzzy control. Moreover, the proposed linear-like synthetical matrix representation and the systematic design procedures might activate a new research direction in the quadratic optimal fuzzy control. Furthermore, the proposed in-depth analysis on the degree of stability and gain margin can provide the researchers with complete perspective of all facets of the resultant closed-loop fuzzy system. Simulation results have manifested that the designed optimal fuzzy controllers can effectively drive the fuzzy system to the target points in short time.

\section{APPENDIX A}

Proof of Theorem 1: Define

$$
\begin{aligned}
\phi^{i}(X(\cdot), R(\cdot)) \triangleq & \int_{t}^{t_{1}^{i}}\left[X^{t}(\tau) L(\tau) X(\tau)\right. \\
& \left.+R^{t}(\tau) W^{t}(Y(\tau)) W(Y(\tau)) R(\tau)\right] d \tau \\
& +X^{t}\left(t_{1}^{i}\right) Q^{i} X\left(t_{1}^{i}\right), \quad t \in\left[t_{0}^{i}, t_{1}^{i}\right]
\end{aligned}
$$

where $X(t)=X^{*}(t)$ is the initial state at time $t$. By the Lagrange multiplier method, we turn the optimal problem into the problem of minimizing

$$
\begin{aligned}
\bar{\phi}^{i}(X(\cdot), R(\cdot)) \\
=\phi^{i}(X(\cdot), R(\cdot)) \\
\quad-2 \int_{t}^{t_{1}^{i}} P^{t}(\tau)[\dot{X}(\tau)-H(X(\tau)) A(\tau) X(\tau) \\
\quad-H(X(\tau)) B(\tau) W(Y(\tau)) R(\tau)] d \tau
\end{aligned}
$$


where $P(\tau) \in \Re^{n}$ is the Langrange multiplier vector. Now, we assume the optimal solutions $\left(X^{*}(\cdot), Y^{*}(\cdot), R^{*}(\cdot)\right)$ exist and, according to the calculus of variations method, let $X(\tau)=$ $X^{*}(\tau)+\epsilon Z(\tau), Y(\tau)=Y^{*}(\tau)+\epsilon \nu(\tau), R(\tau)=R^{*}(\tau)+$ $\epsilon V(\tau), \tau \in\left[t, t_{1}^{i}\right]$, where $V(\tau) \in \Re^{m \delta}$ is the perturbation vector with respect to $R(\tau)$ and $Z(t)=0$ since the initial state at time $t$ is $X(t)=X^{*}(t)$. Then, substituting these variables into (76) and assuming Assumption 1 holds we can obtain

$$
\begin{aligned}
\overline{\phi^{i}}(X(\cdot), R(\cdot))= & \overline{\phi^{i}}\left(X^{*}(\cdot), R^{*}(\cdot)\right)+\epsilon^{2} \phi^{i}(Z(\cdot), V(\cdot)) \\
& +2 \epsilon \int_{t}^{t_{1}^{i}}\left\{Z^{t} L X^{*}+V^{t} W^{t} W R^{*}-P^{t} \dot{Z}\right. \\
& \left.+P^{t} H A Z+P^{t} H B W V\right\} d \tau \\
& +2 \epsilon Z_{t_{1}^{i}}^{t} Q^{i} X_{t_{1}^{i}}^{*} .
\end{aligned}
$$

Hence, the necessary condition for optimality is

$$
\begin{aligned}
& \int_{t}^{t_{1}^{i}}\left\{Z^{t} L X^{*}-P^{t} \dot{Z}+P^{t} H A Z\right\} d \tau \\
& \quad+\int_{t}^{t_{1}^{i}}\left\{V^{t} W^{t} W R^{*}+P^{t} H B W V\right\} d \tau+Z_{t_{1}^{i}}^{t} Q^{i} X_{t_{1}^{i}}^{*}=0 .
\end{aligned}
$$

However, $\quad \int_{t}^{t_{1}^{i}} P^{t}(\tau) \dot{Z}(\tau) d \tau=\left.P^{t}(\tau) Z(\tau)\right|_{t} ^{t_{1}^{i}}-$ $\int_{t}^{t_{1}^{i}} \dot{P}^{t}(\tau) Z(\tau) d \tau$, so (77) becomes

$$
\begin{gathered}
\int_{t}^{t_{1}^{i}} V^{t}\left[W^{t} W R^{*}+W^{t} B^{t} H^{t} P\right] d \tau \\
\quad+\int_{t}^{t_{1}^{i}}\left[L X^{*}+A^{t} H^{t} P+\dot{P}\right]^{t} Z d \tau \\
\quad+Z_{t_{1}^{i}}^{t}\left[Q^{i} X_{t_{1}^{i}}^{*}-P\left(t_{1}^{i}\right)\right]=0 .
\end{gathered}
$$

Since $Z(\cdot)$ and $V(\cdot)$ are independent, we obtain the global minimizer $u^{*}(t)$ in (12), and the corresponding optimal control law $R^{*}(t)$ in (11), where $P(t)$ and the optimal trajectory $X^{*}(t)$ satisfy (13) with $X\left(t_{0}^{i}\right)=X_{0}^{i}$ and $P\left(t_{1}^{i}\right)=Q^{i} X_{t_{1}^{i}}^{*}$.

Proof of Lemma 1: Since, by omitting the explicit timedependence for notation simplification

$$
\begin{aligned}
& \min _{u_{\left[t_{0}, t_{1}\right]}} \int_{t_{0}}^{t_{1}}\left(X^{t} L X+u^{t} u\right) d t+X^{t}\left(t_{1}\right) Q X\left(t_{1}\right) \\
& F=\min _{u_{\left[t_{1}^{1}, t_{1}\right]}}\left\{\operatorname { m i n } _ { u _ { [ t _ { 0 } , t _ { 1 } ^ { 1 } ] } } \left[\int_{t_{0}}^{t_{1}^{1}}\left(X^{t} L X+u^{t} u\right) d t\right.\right. \\
& \left.\left.\quad+\int_{t_{1}^{1}}^{t_{1}}\left(X^{t} L X+u^{t} u\right) d t+X^{t}\left(t_{1}\right) Q X\left(t_{1}\right)\right]\right\} \\
& =\min _{u_{\left[t_{0}^{2}, t_{1}\right]}}\left\{\int_{t_{0}^{2}}^{t_{1}}\left(X^{t} L X+u^{t} u\right) d t+X^{t}\left(t_{1}\right) Q X\left(t_{1}\right)\right. \\
& \left.\quad+\min _{u_{\left[t_{0}, t_{1}^{1}\right]}} \int_{t_{0}}^{t_{1}^{1}}\left(X^{t} L X+u^{t} u\right) d t\right\}
\end{aligned}
$$

$$
\begin{aligned}
= & \min _{u_{\left[t_{0}^{N}, t_{1}\right]}}\left\{\int_{t_{0}^{N}}^{t_{1}}\left(X^{t} L X+u^{t} u\right) d t+X^{t}\left(t_{1}\right) Q X\left(t_{1}\right)\right. \\
& +\min _{u_{\left[t_{0}^{N-1}, t_{1}^{N-1}\right]}}\left[\int_{t_{0}^{N-1}}^{t_{1}^{N-1}}\left(X^{t} L X+u^{t} u\right) d t\right. \\
& +\min _{u_{\left[t_{0}^{N-2}, t_{1}^{N-2}\right]}}\left(\int_{t_{0}^{N-2}}^{t_{1}^{N-2}}\left(X^{t} L X+u^{t} u\right) d t\right. \\
& +\cdots+\min _{u_{\left[t_{0}^{2}, t_{1}^{2}\right]}}\left[\int_{t_{0}^{2}}^{t_{1}^{2}}\left(X^{t} L X+u^{t} u\right) d t\right. \\
& \left.\left.\left.\left.+\min _{u_{\left[t_{0}, t_{1}^{1}\right]}} \int_{t_{0}}^{t_{1}^{t}}\left(X^{t} L X+u^{t} u\right) d t\right] \cdots\right)\right]\right\}
\end{aligned}
$$

we obtain (9) with $X\left(t_{0}^{1}\right)=X_{0}$ and $X\left(t_{0}^{i}\right)=X^{*}\left(t_{1}^{i-1}\right), i=$ $2, \ldots, N$.

Proof of Lemma 3:

2) Omitting the explicit time- and state-dependence for notation simplification and using the above result, we obtain $\left[\lambda I_{n}-H A H B\right]=\left[\sum_{i=1}^{r} h_{i}\left[\lambda I_{n}-A_{i} B_{i}\right]\right]$. Let $\left[\lambda I_{n}-A_{i} B_{i}\right]=\left[a_{1}^{i} a_{2}^{i} \ldots a_{n}^{i} b_{1}^{i} b_{2}^{i} \ldots b_{m}^{i}\right]$, where $a_{j}^{i}$, $j=1, \ldots, n$, and $b_{j}^{i}, j=1, \ldots, m$ are all $n \times 1$ column vectors. Then we have

$\left[\lambda I_{n}-H A H B\right]=\sum_{i=1}^{r} h_{i}\left[a_{1}^{i} a_{2}^{i} \ldots a_{n}^{i} b_{1}^{i} b_{2}^{i} \ldots b_{m}^{i}\right]$.

3) Necessity. Let $\left(A_{i}, B_{i}\right)$ be CC for all $i=1, \ldots, r$. We now assume $\operatorname{rank}\left[\lambda I_{n}-H A H B\right]<n$, which means that at least $m+2$, saying the first $m+2$, column vectors of $\left[\lambda I_{n}-H A H B\right]$ are dependent. Therefore, there exists scalars $l_{i}, i=1, \ldots, m+2$ not all zeros such that

$l_{1}\left(\sum_{i=1}^{r} h_{i} a_{1}^{i}\right)+\cdots+l_{m+2}\left(\sum_{i=1}^{r} h_{i} a_{m+2}^{i}\right)=0$

where $h_{i} \in[0,1]$. Consequently

$$
\sum_{i=1}^{r} h_{i}\left[l_{1} a_{1}^{i}+\cdots+l_{m+2} a_{m+2}^{i}\right]=0 .
$$

In order to guarantee the existence of the equality under all $h_{i}(X(k)) \in[0,1]$, we conclude

$$
l_{1} a_{1}^{i}+\cdots+l_{m+2} a_{m+2}^{i}=0, \quad \forall i=1, \ldots, r .
$$

Hence, $a_{j}^{i}, j=1, \ldots, m+2$, are dependent since $l_{j}, j=$ $1, \ldots, m+2$ are not all zeros. In other words, $\operatorname{rank}\left[\lambda I_{n}-\right.$ $\left.A_{i} B_{i}\right]<n$, for all $i=1, \ldots, r$. Accordingly, $\left(A_{i}, B_{i}\right)$ is not CC for all $i=1, \ldots, r$. This conflicts with the precondition that $\left(A_{i}, B_{i}\right)$ is $\mathrm{CC}$ for all $i=1, \ldots, r$. Therefore, we have proved that $\operatorname{rank}\left[\lambda I_{n}-H A H B\right]=$ $n$.

4) Sufficiency. Reversing the argument in part (2), we can prove that if $\operatorname{rank}\left[\lambda I_{n}-H A H B\right]=n$, then $\left(A_{i}, B_{i}\right)$ is $\mathrm{CC}$, for all $i=1, \ldots, r$. 
Proof of Theorem 5: Since the firing-strength matrix $H(X(t))$ is function of state variables, the general approach to getting a backward Riccati-like DE is not implementable now. Therefore, we define

$$
\begin{aligned}
\Psi\left(X_{t}^{*}, t\right) \triangleq & \min _{R_{\left[t_{0}, t\right]}}\left\{\int_{t_{0}}^{t}\left[X^{t}(\tau) L X(\tau)+u^{t}(\tau) u(\tau)\right] d \tau\right\} \\
= & \min _{R_{\left[t_{0}, t\right]}}\left\{\int_{t-\xi}^{t}\left[X^{t}(\tau) L X(\tau)+u^{t}(\tau) u(\tau)\right] d \tau\right. \\
& \left.+\Psi\left(X_{t-\xi}^{*}, t-\xi\right)\right\}
\end{aligned}
$$

where $R^{t}(\cdot) W^{t}(Y(\cdot)) W(Y(\cdot)) R(\cdot)$ is denoted by $u^{t}(\cdot) u(\cdot)$ and $L(\cdot)$ by $L$ for notation simplification. Also, we have

$$
\begin{aligned}
& \int_{t-\xi}^{t}\left[X^{t}(\tau) L X(\tau)+u^{t}(\tau) u(\tau)\right] d \tau \\
& \quad=\left[X_{t}^{t} L X_{t}+R_{t}^{t} W^{t}\left(Y_{t}\right) W\left(Y_{t}\right) R_{t}\right](-\xi)+O_{1}\left(\xi^{2}\right) \\
& \Psi\left(X_{t-\xi}^{*}, t-\xi\right)=\Psi\left(X_{t}^{*}, t\right)+\left[\nabla_{X} \Psi\left(X_{t}^{*}, t\right)\right]^{t}\left(X_{t-\xi}^{*}-X_{t}^{*}\right) \\
& \quad+\frac{\partial \Psi}{\partial t}\left(X_{t}^{*}, t\right)(-\xi)+O_{2}\left(\xi^{2}\right) \\
& \quad X_{t}^{*}-X_{t-\xi}^{*}=\left[H\left(X_{t}^{*}\right) A(t) X_{t}^{*}\right. \\
& \left.\quad+H\left(X_{t}^{*}\right) B(t) W\left(Y_{t}^{*}\right) R_{t}\right] \xi+O_{3}\left(\xi^{2}\right)
\end{aligned}
$$

where $O_{i}\left(\xi^{2}\right)$ and $i=1,2,3$ denote the polynomials of $\xi^{2}$. Therefore, by substituting (79)-(81) into (78), we obtain, by setting $\xi \rightarrow 0$

$$
\begin{aligned}
\frac{\partial \Psi}{\partial t}\left(X_{t}^{*}, t\right) & \min _{R_{t} \in L_{2}\left(t_{0}, \infty ; R^{m \delta}\right)}\left\{X_{t}^{t} L X_{t}+R_{t}^{t} W\left(Y_{t}\right)^{t} W\left(Y_{t}\right) R_{t}\right. \\
& -\left[\nabla_{X} \Psi\left(X_{t}^{*}, t\right)\right]^{t}\left[H\left(X_{t}^{*}\right) A(t) X_{t}^{*}\right. \\
& \left.\left.+H\left(X_{t}^{*}\right) B(t) W\left(Y_{t}^{*}\right) R_{t}\right]\right\} .
\end{aligned}
$$

Minimizing the right-hand side of the above equation with respect to $R_{t}$, we obtain the optimal synthetical control law

$$
\begin{aligned}
R_{t}^{*}= & \frac{1}{2} W^{t}\left(Y_{t}^{*}\right)\left[W\left(Y_{t}^{*}\right) W^{t}\left(Y_{t}^{*}\right)\right]^{-1} \\
& \times B^{t}(t) H^{t}\left(X_{t}^{*}\right) \nabla_{X} \Psi\left(X_{t}^{*}, t\right)
\end{aligned}
$$

with $\Psi\left(X_{t_{0}}^{*}, t_{0}\right)=0$. We may assume the solution is of the form $\Psi\left(X_{t}^{*}, t\right)=X_{t}^{*^{t}} K(t) X_{t}^{*}$ with $K\left(t_{0}\right)=0$, where $K(t)$ is the introduced $n \times n$ symmetric positive semidefinite time variable matrix. Hence, we have

$$
\begin{aligned}
X_{t}^{*^{t}} \dot{K}(t) X_{t}^{*}= & X_{t}^{*^{t}} L X_{t}^{*}-X_{t}^{*^{t}} H\left(X_{t}^{*}\right) B B^{t} H^{t}\left(X_{t}^{*}\right) X_{t}^{*} \\
& -2 X_{t}^{*^{t}} K(t) H\left(X_{t}^{*}\right) A X_{t}^{*}
\end{aligned}
$$

and then we get all the results.

\section{REFERENCES}

[1] H. Wang, K. Tanaka, and M. Griffin, "Parallel distributed compensation of nonlinear systems by Takagi and Sugeno's fuzzy model," in Proc. FUZZ-IEEE'95, Yokohama, Japan, Mar. 1995, pp. 531-538.

[2] H. O. Wang, K. Tanaka, and M. F. Griffin, "An approach to fuzzy control of nonlinear system: Stability and design issues," IEEE Trans. Fuzzy Syst., vol. 4, pp. 14-23, Feb. 1996.

[3] K. Tanaka, T. Ikeda, and H. O. Wang, "Robust stabilization of a class of uncertain nonlinear systems via fuzzy control: Quadratic stabilizability, $H^{\infty}$ control theory and linear matrix inequalities," IEEE Trans. Fuzzy Syst., vol. 4, pp. 1-13, Feb. 1996.

[4] H. O. Wang and K. Tanaka, "An LMI-based stable fuzzy control of nonlinear systems and its application to control of chaos," in Proc. FUZZIEEE'96, vol. 2, New Orleans, LA, Sept. 1996, pp. 1433-1438.

[5] K. Tanaka and H. O. Wang, Fuzzy Control Systems Analysis and Design: A Linear Matrix Inequality Approach. New York: Wiley, 2000.

[6] K. Tanaka and T. Kosaki, "Design of a stable fuzzy controller for an articulated vehicle," IEEE Trans. Syst., Man, Cybern., pt. B, vol. 27, pp. 552-558, June 1997.

[7] X. J. Ma, Z. Q. Sun, and Y. Y. He, "Analysis and design of fuzzy controller and fuzzy observer," IEEE Trans. Fuzzy Syst., vol. 6, pp. 41-51, Feb. 1998.

[8] S. G. Cao, N. W. Rees, and G. Feng, "Analysis and design for a class of complex control systems-Part I: Fuzzy modeling and identification," Automatica, vol. 33, no. 6, pp. 1017-1028, 1997.

[9] - "Analysis and design for a class of complex control systems, part II: Fuzzy controller design," Automatica, vol. 33, no. 6, pp. 1029-1039, 1997.

[10] L. X. Wang, "Stable and optimal fuzzy control of linear systems," IEEE Trans. Fuzzy Syst., vol. 6, pp. 137-143, Feb. 1998

[11] K. Tanaka and M. Sano, "Frequency shaping for fuzzy control systems with unkown nonlinear plants by a learning methods of neural network," Fuzzy Sets Syst., vol. 71, pp. 71-84, 1995.

[12] H. K. Lam, F. H. F. Leung, and P. K. S. Tam, "Stable and robust fuzzy control for uncertain nonlinear systems based on a grid-point approach," in Proc. FUZZ-IEEE'97, vol. 1, Barcelona, Spain, July 1997, pp. 281-285.

[13] H. J. Kang and C. Kwon, " $L^{\infty}$ robust stability analysis for the fuzzy feedback linearization regulator," in Proc. FUZZ-IEEE'97, vol. 1, Barcelona, Spain, July 1997, pp. 277-280.

[14] K. S. Ray and D. D. Maunder, "Application of the circle criteria for stability analysis of linear SISO and MIMO systems associated with fuzzy logic controllers," IEEE Trans. Syst., Man, Cybern., vol. SMC-14, pp. 345-349, Mar./Apr. 1984.

[15] L. X. Wang, "A supervisory controller for fuzzy control systems that guarantees stability," in Proc. FUZZ-IEEE'94, Orlando, FL, June 1994, pp. 1035-1039.

[16] K. Tanaka, T. Taniguchi, and H. O. Wang, "Fuzzy control based on quadratic performance function," in Proc. 37th IEEE Conf. Decision Contr., Tampa, FL, Dec. 1998, pp. 2914-2919.

[17] — - "Model-based fuzzy control of TORA system: Fuzzy regulator and fuzzy observer design via LMI's that reprensent decay rate, disturbance rejection, robustness, optimality," in Proc. FUZZ-IEEE'98, Anchorage, AK, May 1998, pp. 313-318

[18] J. Douglas, Collocation Methods for Parabolic Equations in a Single Space Variable: Based in C'-Piecewise-Polynomial Spaces. New York: Springer-Verlag, 1974.

[19] B. D. O. Anderson, Linear Optimal Control. Englewood Cliffs, NJ: Prentice-Hall, 1971.

[20] B. D. O. Anderson and J. B. Moore, Optimal Control: Linear Quadratic Methods. Englewood Cliffs, NJ: Prentice-Hall, 1990.

[21] J. Macki and A. Strauss, Introduction to Optimal Control Theory. New York, NY: Springer-Verlag, 1982.

[22] F. M. Callier and C. A. Desoer, Linear System Theory. New York: Springer-Verlag, 1991

[23] R. E. Kalman, "When is a linear control system optimal?," Trans. ASME Ser. D-J. Basic Eng., vol. 86, pp. 1-10, Mar. 1964.

[24] B. D. O. Anderson and J. B. Moore, "Linear system optimization with prescribed degree of stability," Proc. Inst. Elect. Eng., vol. 116, no. 12, pp. 2083-2087, 1965.

[25] S. J. Wu, Linear optimal control. Lecture Notes, by Prof. P. K. C. Wang, EE240B, Univ. California, Los Angeles.

[26] R. W. Brockett, Finite-Dimensional Linear Systems. New York: Wiley, 1970 . 


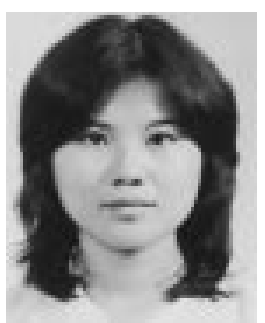

Shinq-Jen Wu received the B.S. degree in chemical engineering from the National Taiwan University, Taipei, Taiwan, R.O.C., in 1986, the M.S. degree in chemical engineering from the National Tsing-Hua University, Hsinchu, Taiwan, in 1989, the M.S. degree in electrical engineering from the University of California, Los Angeles, in 1994, and the Ph.D. degree in electrical engineering from the National Chiao-Tung University, Hsinchu, Taiwan, in 2000.

From September 1989 to July 1990, she was with the Laboratory for Simulation and Control Technology, Chemical Engineering Division of Industrial Technology Research Institute, Hsinchu, Taiwan. From 1990 to 1991, she was with the Chemical Engineering Department, Kao-Yuan Junior College of Technology and Commerce, Kaohsiung, Taiwan. From 1995 to 1996, she was an engineer with the Integration Engineering Department, Macronix International Co., Ltd., Hsinchu, Taiwan. She is now with the Electrical Engineering Department, Da-Yeh University, Chang-Hwa, Taiwan. Her research interests include thermodynamics, transport phenomena, process control and design, especially in the VLSI, biomedical, and petroleum industries, system and control theory, especially in optimal control, filtering theory, fuzzy system theory, and optimal fuzzy controller and tracker design.

Dr. Wu is a member of the Phi Tau Phi Scholastic Honor Society.

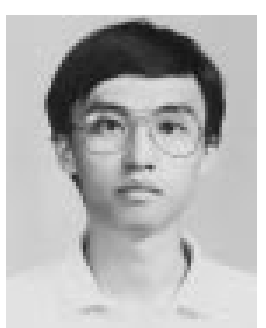

Chin-Teng Lin (S'88-M'91-SM'99) received the B.S. degree in control engineering from the National Chiao-Tung University, Hsinchu, Taiwan, R.O.C., in 1986, and the M.S.E.E. and Ph.D. degrees in electrical engineering from Purdue University, West Lafayette, IN, in 1989 and 1992, respectively.

Since August 1992, he has been with the College of Electrical Engineering and Computer Science, National Chiao-Tung University, Hsinchu, Taiwan, where he is currently a Professor of Electrical and Control Engineering. He also serves as the Deputy Dean of the Research and Development Office of the National Chiao-Tung University since 1998. He is the coauthor of Neural Fuzzy Systems-A Neuro-Fuzzy Synergism to Intelligent Systems (Englewood Cliffs, NJ: Prentice-Hall, 1996) and the author of Neural Fuzzy Control Systems with Structure and Parameter Learning (Singapore: World Scientific, 1994). He has published over 50 journal papers in the areas of neural networks and fuzzy systems. His current research interests include fuzzy systems, neural networks, intelligent control, human-machine interface, and video and audio processing.

Dr. Lin is a member of Tau Beta Pi and Eta Kappa Nu. He is also a member of the IEEE Computer Society, the IEEE Robotics and Automation Society, and the IEEE Systems, Man, and Cybernetics Society. He has been the Executive Council Member of Chinese Fuzzy System Association (CFSA) since 1995, and the Supervisor of Chinese Automation Association since 1998. He was the Vice Chairman of IEEE Robotics and Automation Taipei Chapter in 1996 and 1997. He won the Outstanding Research Award granted by National Science Council (NSC), Taiwan, from 1997 to 1998 and from 1999 to 2000, the Outstanding Electrical Engineering Professor Award from the Chinese Institute of Electrical Engineering (CIEE) in 1997, and the Outstanding Engineering Professor Award from the Chinese Institute of Engineering in 2000. 